\title{
Heavy quarkonia in a bulk viscous medium
}

\author{
Lata Thakur, ${ }^{a}$ Najmul Haque ${ }^{b}$ and Yuji Hirono ${ }^{a, c}$ \\ ${ }^{a}$ Asia Pacific Center for Theoretical Physics, \\ Pohang, Gyeongbuk 37673, Republic of Korea \\ ${ }^{b}$ School of Physical Sciences, National Institute of Science Education and Research, \\ HBNI, Jatni 752050, India \\ ${ }^{c}$ Department of Physics, POSTECH, \\ Pohang, Gyeongbuk 37673, Republic of Korea \\ E-mail: lata.thakur@apctp.org, nhaque@niser.ac.in, \\ yuji.hirono@gmail.com
}

ABSTRACT: We study the properties of heavy quarkonia in a quark gluon plasma in the presence of bulk viscous effects. Within the hard thermal loop approximation at one-loop, the dielectric permittivity of a quark gluon plasma is computed, where the bulk viscous effect enters through the deformation of the distribution functions of thermal quarks and gluons. Based on the modified dielectric permittivity, we compute the in-medium heavy quark potential, that includes non-pertubative string-like terms as well as the perturbative Coulombic term. We discuss how the bulk viscous effect modify the real and imaginary parts of the in-medium potential. Several prescriptions are examined as to how to include the string-like non-perturbative potentials. Using the deformed potential, we compute the wave functions, binding energies, and decay widths of heavy quarkonia in a bulk viscous medium, and study their sensitivity to the strength of the bulk viscous effect. An estimate of the melting temperatures is given.

Keywords: Perturbative QCD, Quark-Gluon Plasma, Resummation

ARXIV EPRINT: 2004.03426 


\section{Contents}

1 Introduction 1

2 Color dielectric permittivity of a bulk viscous medium 2

2.1 Computation of the dielectric permittivity 3

2.1.1 Retarded propagator 3

2.1.2 Symmetric propagator 5

2.1.3 Dielectric permittivity 7

$\begin{array}{lll}2.2 & \text { Two Debye masses and a modified fluctuation-dissipation theorem } & 7\end{array}$

3 In-medium potentials in the presence of bulk viscous corrections $\quad 8$

$\begin{array}{ll}3.1 \text { Approach based on the linear response } & 9\end{array}$

$\begin{array}{ll}3.2 & \text { Introduction of non-perturbative propagator } \\ 3.31\end{array}$

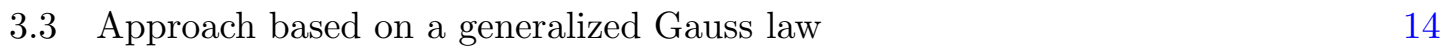

$\begin{array}{ll}3.4 & \text { Comparison of the real part of the potentials } \\ \end{array}$

4 Heavy quarkonia in the presence of bulk viscous corrections $\quad 15$

$\begin{array}{ll}4.1 \text { Computational setup } & 15\end{array}$

$\begin{array}{ll}4.2 \text { Wave function } & 17\end{array}$

$\begin{array}{ll}\text { 4.3 Binding energies and decay widths } & 18\end{array}$

$\begin{array}{lll}\text { 4.3.1 Dependence on the scale } \Lambda & 18\end{array}$

$\begin{array}{lll}\text { 4.3.2 Effect of bulk viscous corrections } & 19\end{array}$

5 Summary and discussions $\quad 22$

\section{Introduction}

The relativistic heavy-ion collisions provide us with a unique opportunity to experimentally study the strongly interacting matter in extreme conditions. The currently ongoing experimental programs at the Relativistic Heavy Ion Collider (RHIC) at BNL and the Large Hadron Collider (LHC) at CERN aim at revealing the properties of the quark gluon plasma (QGP), which is expected to appear at high temperatures. At sufficiently high temperatures, a QGP behaves as a weakly interacting gas of quarks and gluons, which can be understood using hard thermal loop (HTL) resummation [1-4]. Such a description has been successful in describing the thermodynamics of the QGP even close to the crossover temperature [5-7].

Heavy quarkonium states have been a useful probe of the surrounding thermal medium. In the vacuum, they are reliably described in terms of non-relativistic potential models $[8,9]$ using the Cornell potential $[10,11]$. A QGP medium exhibits the screening of static colorelectric fields and that would result in the melting of heavy quarkonia, which was one of the first proposed signals of the formation of a QGP [12]. The potential models have been applied to the study of quarkonia at finite temperatures, the first of such works is done 
by Karsch, Mehr, and Satz [13]. The meson current correlators and quarkonium spectral functions have been calculated from potential models [14-21] and are compared to the first-principle lattice QCD calculations [22-30]. The appearance of the imaginary part of the potential due do the Landau damping [31-34] and the break up of a color singlet bound state into a color octet quark-antiquark state via absorption of a thermal gluon is discussed [35-37], which has further stimulated the study of complex heavy quarkonia potential from thermal field theories [38-41] as well as from the lattice QCD [42-44]. See ref. [45] for a recent review.

The motivation for the current work is to understand how the effect of non-equilibrium nature of the fluid is imprinted on the properties of heavy quarkonia. For example, in the early time of a relativistic heavy-ion collision, the longitudinal expansion is stronger than the radial expansion, which would result in an anisotropy of the distribution functions of medium particles in the momentum space. The effect of such momentum-space anisotropies on quarkonia has been discussed in refs. [38, 40, 41, 46-52]. The presence of magnetic fields [53-61] or non-zero fluid velocity [62-69] also works as a source of anisotropy. Among such non-equilibrium situations, the role the bulk viscosity is gaining an increasing attention in relation to the beam energy scan program [70], since the bulk viscous effect is expected to be enhanced as the system approaches a critical point [71-73].

The goal of this study is to test the sensitivity of quarkonia to the non-equilibrium nature of the fluid, in particular, the bulk viscous corrections. In ref. [74], the color dielectric permittivity is computed in the presence of bulk viscous corrections based on the HTL-resummed gluon propagators. The bulk viscous effect enters through the deformation of the distribution functions of thermal particles. The perturbative HTL gluon propagators only gives rise to the Coulombic potential, but non-perturbative string-like contributions have been observed in lattice QCD studies [75-77]. There has been several proposed prescriptions as to how to incorporate non-perturbative contributions in the potential $[44,49,69,78,79]$. Among those is an approach based on the linear response theory: the modified string-like potential is obtained by modifying the linear potential using the HTL permittivity that entails the medium effect. ${ }^{1}$ In this work, based on the modified dielectric permittivity in the presence of bulk viscous effect, we derive a complex heavy quark potential in such environments. We examine several prescriptions for the introduction of non-perturbative part. We use the modified potential to solve the Schrödinger equation and compute the deformed wave functions, binding energies, and decay widths of heavy quarkonia. We discuss how those physical properties are affected by the bulk viscous effect.

The rest of the article is structured as follows. In section 2, we derive the dielectric permittivity of a thermal medium in the presence of bulk viscous corrections. In section 3, we calculate the complex heavy quark potential based on the modified dielectric permittivity and discuss its properties. In section 4, we show the effect of the bulk viscous corrections on the binding energies and decay widths of quarkonium states, from which melting temperatures are estimated. Section 5 is devoted to summary and discussions.

\section{Color dielectric permittivity of a bulk viscous medium}

In order to compute the in-medium potential, we rely on the linear response theory, in which the in-medium properties are encoded in the color dielectric permittivity. We here review

\footnotetext{
${ }^{1}$ This approach is taken in refs. $[41,49,78]$ to study heavy quarkonia in an anisotropic medium.
} 
the derivation of the dielectric permittivity in the HTL approximation in the presence of the bulk viscous correction. When the system is away from thermal equilibrium, the fluctuation-dissipation theorem is violated, which leads to the existence of two different Debye masses. In the current situation, a modified fluctuation-dissipation theorem is found to hold.

In the computation below, the non-equilibrium effect enters through the modification of the distribution function of thermal quarks and gluons,

$$
f(\mathbf{k})=f_{0}(k)+\delta_{\text {noneq }} f(\mathbf{k}),
$$

where $f_{0}(k)$ is the equilibrium distribution, ${ }^{2}$ and the second term is the non-equilibrium correction. In general, non-equilibrium corrections can be anisotropic. Such an anisotropy may be present at the early stage of heavy-ion collisions, where the longitudinal expansion is substantially stronger than the radial expansion. Certain types of the corrections can be regarded as viscous corrections when the anisotropy is weak. In this study, we discuss the effect of the bulk viscosity, and as $f_{0}$ we take the thermal equilibrium one,

$$
f(\mathbf{k})=f_{0}(k)+\delta_{\text {bulk }} f(k),
$$

where the correction $\delta_{\text {bulk }} f(k)$ is isotropic. The specific form of the correction is given later in eq. (2.7)

\subsection{Computation of the dielectric permittivity}

Let us here derive the dielectric permittivity in the presence of bulk viscous corrections. For this, we compute the gluon self-energies and propagators in the presence of bulk viscous corrections. In the following, we review how to obtain the modified propagators as done in ref. [74]. The medium quarks are taken to be massless.

\subsubsection{Retarded propagator}

First, let us look at the retarded self-energy of gluons. In the following computations, we employ the Coulomb gauge. To evaluate the potential in the Coulomb gauge, we need the temporal component of the self energy $\Pi_{R}(P) \equiv \Pi_{R}^{00}(P) \cdot{ }^{3}$ In the HTL approximation, the one-loop contribution from $N_{f}$ quarks to $\Pi_{R}(P)$ is given by $[74,80]$

$$
\Pi_{R}^{(q)}(P)=\frac{4 \pi N_{f} g^{2}}{(2 \pi)^{4}} \int k d k d \Omega\left(\frac{f^{+}(\mathbf{k})+f^{-}(\mathbf{k})}{2}\right) \frac{1-(\hat{\mathbf{k}} \cdot \hat{\mathbf{p}})^{2}}{\left(\hat{\mathbf{k}} \cdot \hat{\mathbf{p}}+\frac{p^{0}+i \epsilon}{p}\right)^{2}},
$$

where $\hat{\mathbf{k}} \equiv \mathbf{k} / k$ and $f^{ \pm}(\mathbf{k})$ are distribution functions for quarks/antiquarks. As long as the HTL approximation is valid, this expression is true even in non-equilibrium situations. In the thermal equilibrium, the distribution functions are given by

$$
f_{0}^{ \pm}(k)=\frac{1}{e^{(k \mp \mu) / T}+1},
$$

where $\mu$ is the quark chemical potential. The contribution from the gluon loop has the same structure with the Fermi distribution replaced with the Bose one. Including the

\footnotetext{
${ }^{2}$ As a reference point, $f_{0}$, one may take a distribution of a non-thermal fixed point.

${ }^{3}$ We denote a four momentum by a capital letter, $P=\left(p^{0}, \mathbf{p}\right)$, and $p \equiv|\mathbf{p}|$.
} 
contribution from quark and gluon loops, the retarded self-energy in the equilibrium is written as

$$
\Pi_{R}^{\mathrm{eq}}(P)=m_{D}^{2}\left(\frac{p^{0}}{2 p} \ln \frac{p^{0}+p+i \epsilon}{p^{0}-p+i \epsilon}-1\right),
$$

where $m_{D}$ is the Debye mass,

$$
m_{D}^{2}=\frac{g^{2} T^{2}}{6}\left[2 N_{c}+N_{f}\left(1+\frac{3 \tilde{\mu}^{2}}{\pi^{2}}\right)\right]
$$

with $\tilde{\mu} \equiv \mu / T$.

Now let us introduce the bulk viscous correction. We shall model the correction with the following form [74],

$$
\delta_{\text {bulk }} f(k)=\left(\frac{k}{T}\right)^{a} \Phi f_{0}(k)\left(1 \pm f_{0}(k)\right),
$$

where $a$ and $\Phi$ are constants and the $+(-)$ sign is for Bose (Fermi) distribution. $\Phi$ is a parameter proportional to the bulk viscous pressure (divided by ideal pressure). There are constraints for the parameter $a$. We need a condition $a>0$ so that there will be no IR divergence coming from the correction in the retarded self-energy of gluons (see eq. (2.3) with $f$ replaced by the Bose distribution). Otherwise the dominant contribution does not come from $k \sim T$ and the HTL approximation becomes invalid. The same condition of the absence of IR divergence for the symmetric self-energy (gluon-loop version of eq. (2.18)) leads to a stronger bound, $a>1 / 2$, from the $\mathcal{O}\left(\Phi^{2}\right)$ contributions. In addition, in order for the bulk viscous contribution to be non-negligible compared to the NLO corrections, we need the condition $|\Phi| \gg g^{2}$. The bulk pressure $\delta_{\text {bulk }} p$ is usually negative, which corresponds to $\Phi<0$, but the sign can be reversed in the presence of shear-bulk coupling [81]. In the present study, we regard $\Phi$ as a parameter of either sign.

In the presence of bulk correction (2.7), the retarded self-energy is modified as $\Pi_{R}=$ $\Pi_{R}^{\mathrm{eq}}+\delta_{\mathrm{bulk}} \Pi_{R}$. The contribution from the quark loop, $\delta_{\mathrm{bulk}} \Pi_{R}^{(q)}$, is given by

$$
\begin{aligned}
\delta_{\text {bulk }} \Pi_{R}^{(q)}(P)= & \frac{N_{f} g^{2}}{(2 \pi)^{3}} \int k d k\left[\delta_{\text {bulk }} f^{+}(k)+\delta_{\text {bulk }} f^{-}(k)\right] \int d \Omega \frac{1-(\hat{\mathbf{k}} \cdot \hat{\mathbf{p}})^{2}}{\left(\hat{\mathbf{k}} \cdot \hat{\mathbf{p}}+\frac{p_{0}+i \epsilon}{p}\right)^{2}}, \\
= & \frac{N_{f} g^{2}}{(2 \pi)^{3}} \int k d k\left(\frac{k}{T}\right)^{a} \Phi\left[f_{0}^{+}(k)\left(1-f_{0}^{+}(k)\right)+f_{0}^{-}(k)\left(1-f_{0}^{-}(k)\right)\right] \\
& \times\left(\frac{p^{0}}{2 p} \ln \frac{p^{0}+p+i \epsilon}{p^{0}-p+i \epsilon}-1\right) .
\end{aligned}
$$

The correction does not affect the momentum dependence and just modify the Debye mass. Similarly, the contribution from the gluon loop can be computed. The total retarded selfenergy including bulk correction can be written as [74]

$$
\begin{aligned}
\Pi_{R}(P) & =\Pi_{R}^{\mathrm{eq}}+\delta_{\mathrm{bulk}} \Pi_{R}^{(q)}+\delta_{\mathrm{bulk}} \Pi_{R}^{(g)} \\
& =\widetilde{m}_{D, R}^{2}\left(\frac{p^{0}}{2 p} \ln \frac{p^{0}+p+i \epsilon}{p^{0}-p+i \epsilon}-1\right),
\end{aligned}
$$

where $\widetilde{m}_{D, R}^{2}=m_{D}^{2}+\delta m_{D, R}^{2}$ is the modified Debye mass. The correction is written as

$$
\delta m_{D, R}^{2}=\frac{g^{2} T^{2}}{6}\left[2 N_{c} c_{R}^{g}(a) \Phi+N_{f}\left(1+\frac{3 \tilde{\mu}^{2}}{\pi^{2}}\right) c_{R}^{q}(a, \tilde{\mu}) \Phi\right] .
$$


Here, the dimensionless quantities $c_{R}^{q}(a, \tilde{\mu})$ and $c_{R}^{g}(a)$ are defined by

$$
c_{R}^{q, g}=\frac{1}{\Phi} \frac{\int k d k \delta_{\mathrm{bulk}} f(k)}{\int k d k f_{0}(k)}
$$

where we take the Bose and Fermi distributions as $f_{0}$ for $c_{R}^{g}(a)$ and $c_{R}^{q}(a, \tilde{\mu})$ respectively, and their explicit forms are

$$
\begin{aligned}
c_{R}^{q}(a, \tilde{\mu}) & =-\frac{6}{\pi^{2}+3 \tilde{\mu}^{2}} \Gamma(a+2)\left[\operatorname{Li}_{a+1}\left(-e^{\tilde{\mu}}\right)+\operatorname{Li}_{a+1}\left(-e^{-\tilde{\mu}}\right)\right], \\
c_{R}^{g}(a) & =\frac{6}{\pi^{2}} \Gamma(a+2) \zeta(a+1),
\end{aligned}
$$

where $\operatorname{Li}_{n}(z)$ denotes the polylogarithm function. At the vanishing quark chemical potential $\mu=0$, the quark part $c_{R}^{q}$ simplify to

$$
c_{R}^{q}(a, \tilde{\mu}=0)=\frac{12}{\pi^{2}}\left(1-2^{-a}\right) \Gamma(a+2) \zeta(a+1) .
$$

We can compute the retarded propagator from the self-energy. In the Coulomb gauge, if the distribution function is isotropic, the temporal component of the resummed propagator. ${ }^{4} \bar{D}_{R}(P) \equiv \bar{D}_{R}^{00}(P)$, is independent of the spatial components of the self-energy and propagators. ${ }^{5}$ The Dyson-Schwinger equation reads

$$
\bar{D}_{R}=D_{R}+D_{R} \Pi_{R} \bar{D}_{R}
$$

where $D_{R}=1 /\left(p^{2}+i \operatorname{sgn}\left(p_{0}\right) \epsilon\right)$ is the bare propagator. Using the $\Pi_{R}(P)$ obtained above, the temporal component of the resummed retarded propagator is written as

$$
\bar{D}_{R}(P)=\frac{1}{p^{2}-\Pi_{R}} .
$$

For the computation of the potential, we need the static limit $p_{0} \rightarrow 0$ of the propagator. To the first order in $p_{0}$, it can be written as

$$
\bar{D}_{R}(P)=\frac{1}{p^{2}+\widetilde{m}_{D, R}^{2}}-i \pi \frac{p_{0}}{2 p} \frac{\widetilde{m}_{D, R}^{2}}{\left(p^{2}+\widetilde{m}_{D, R}^{2}\right)^{2}} \Theta\left(p^{2}-p_{0}^{2}\right)+O\left(p_{0}^{2}\right),
$$

where $\Theta$ is the step function. The advanced propagator is given by the complex conjugate of the retarded one.

\subsubsection{Symmetric propagator}

The symmetric propagator $D_{S}(P)$ and the self-energy $\Pi_{S}(P)$ can be computed in a similar manner. The quark loop contribution to $\Pi_{S}(P)$ is written as

$$
\Pi_{S}^{(q)}(P)=4 i N_{f} g^{2} \pi^{2} \int \frac{k^{2} d k}{(2 \pi)^{3}} \sum_{i= \pm} f^{i}(k)\left(f^{i}(k)-1\right) \frac{2}{p} \Theta\left(p^{2}-p_{0}^{2}\right) .
$$

\footnotetext{
${ }^{4}$ Resummed propagators are indicated by characters with bars.

${ }^{5}$ In the Coulomb gauge, the bare and resummed propagators satisfy the condition,

$$
p_{i} D_{R}^{0 i}=0 .
$$

When the system is isotropic, like in the current case, we have $D_{R}^{0 i}=0$.
} 
Adding the quark-loop and gluon-loop contributions, the equilibrium part of $\Pi_{S}(P)$ is written using the Debye mass (2.6) as

$$
\Pi_{S}^{\mathrm{eq}}(P)=-2 \pi i m_{D}^{2} \frac{T}{p} \Theta\left(p^{2}-p_{0}^{2}\right) .
$$

The total symmetric self-energy with the bulk viscous correction is again represented with the modified Debye mass,

$$
\begin{aligned}
\Pi_{S}(P) & =\Pi_{S}^{\mathrm{eq}}+\delta_{\mathrm{bulk}} \Pi_{S} \\
& =-2 \pi i \widetilde{m}_{D, S}^{2} \frac{T}{p} \Theta\left(p^{2}-p_{0}^{2}\right),
\end{aligned}
$$

where $\widetilde{m}_{D, S}^{2}=m_{D}^{2}+\delta m_{D, S}^{2}$, and the bulk viscous correction $\delta m_{D, S}^{2}$ is given to the first order in $\Phi$ by

$$
\delta m_{D, S}^{2}=\frac{g^{2} T^{2}}{6}\left(2 N_{c} c_{S}^{g}(a) \Phi+N_{f}\left(1+\frac{3 \tilde{\mu}^{2}}{\pi^{2}}\right) c_{S}^{q}(a, \tilde{\mu}) \Phi\right)
$$

where the dimensionless functions $c_{S}^{q}(a, \tilde{\mu})$ and $c_{S}^{g}(a)$ are defined as

$$
c_{S}^{q, g}=\frac{1}{\Phi} \frac{\int d k k^{2} \delta_{\mathrm{bulk}} f(k)\left(1 \pm 2 f_{0}(k)\right)}{\int d k k^{2} f_{0}(k)\left(1 \pm f_{0}(k)\right)}
$$

whose explicit forms are

$$
\begin{aligned}
c_{S}^{q}(a, \tilde{\mu}) & =-\frac{3}{\pi^{2}+3 \tilde{\mu}^{2}} \Gamma(a+3)\left[\operatorname{Li}_{a+1}\left(-e^{-\tilde{\mu}}\right)+\operatorname{Li}_{a+1}\left(-e^{\tilde{\mu}}\right)\right], \\
c_{S}^{g}(a) & =\frac{3}{\pi^{2}} \Gamma(a+3) \zeta(a+1) .
\end{aligned}
$$

Given the symmetric self-energy, we can compute the symmetric propagator. The temporal component of the resummed symmetric propagator in the Coulomb gauge satisfies the following Dyson-Schwinger equation

$$
\bar{D}_{S}=D_{S}+D_{R} \Pi_{R} \bar{D}_{S}+D_{S} \Pi_{A} \bar{D}_{A}+D_{R} \Pi_{S} \bar{D}_{A}
$$

Using the Dyson-Schwinger equation for the retarded and advanced propagators, $\bar{D}_{R}=$ $D_{R}+D_{R} \Pi_{R} \bar{D}_{R}, \bar{D}_{A}=D_{A}+D_{A} \Pi_{A} \bar{D}_{A}$, we can write eq. (2.25) in the following form,

$$
\left(\bar{D}_{R}\right)^{-1} \bar{D}_{S}\left(\bar{D}_{A}\right)^{-1}=\left(D_{R}\right)^{-1} D_{S}\left(D_{A}\right)^{-1}+\Pi_{S} .
$$

The first term on the right hand side is in fact zero (note that it is proportional to $p^{4} \delta\left(p^{2}\right)$ ). Thus, the resummed symmetric propagator can be written as $\bar{D}_{S}=\bar{D}_{R} \Pi_{S} \bar{D}_{A}$ and in $p_{0} \rightarrow 0$ limit it is given by

$$
\bar{D}_{S}=-\frac{2 \pi i T \widetilde{m}_{D, S}^{2}}{p\left(p^{2}+\widetilde{m}_{D, R}^{2}\right)^{2}},
$$

where we have used eqs. (2.9) and (2.17). 


\subsubsection{Dielectric permittivity}

The dielectric permittivity, $\varepsilon(p)$, is computed as

$$
\varepsilon^{-1}(p)=\lim _{p^{0} \rightarrow 0} p^{2} \bar{D}_{11}(P),
$$

where $\bar{D}_{11}(P)$ is the longitudinal component of the 11-part of the resummed gluon propagator. Noting that

$$
\bar{D}_{11}=\frac{1}{2}\left(\bar{D}_{R}+\bar{D}_{A}+\bar{D}_{S}\right),
$$

and using eq. (2.17) and eq. (2.27), we obtain the expression for the dielectric permittivity in the presence of bulk viscous correction,

$$
\varepsilon^{-1}(p)=\frac{p^{2}}{p^{2}+\widetilde{m}_{D, R}^{2}}-i \frac{\pi T p \widetilde{m}_{D, S}^{2}}{\left(p^{2}+\widetilde{m}_{D, R}^{2}\right)^{2}} .
$$

In the limit of vanishing bulk correction, both of $\widetilde{m}_{D, R}$ and $\widetilde{m}_{D, S}$ approaches $m_{D}$ and the equilibrium expression is reproduced. The dielectric permittivity (2.30) will be used in computing the in-medium heavy quarkonia potential.

\subsection{Two Debye masses and a modified fluctuation-dissipation theorem}

We have learned that the effects of the bulk viscous correction are incorporated in two different Debye masses, $\widetilde{m}_{D, R}^{2}$ and $\widetilde{m}_{D, S}^{2}$, that characterize the retarded (advanced) propagators and the symmetric propagator. The two Debye masses are functions of the bulk viscous correction parameter $\Phi$. We have obtained the expression of the modified dielectric permittivity (2.30), which is the main result of this section.

Let us show the behaviors of the modified Debye masses. The bulk viscous correction can be written as

$$
\frac{\widetilde{m}_{D, R(S)}^{2}}{m_{D}^{2}}=1+c_{R(S)}(a, \tilde{\mu}) \Phi .
$$

The expression of $c_{R}$ and $c_{S}$ follows from eqs. (2.10) and (2.21). In figure 1, we show the linear coefficients $c_{R}(a, \tilde{\mu})$ and $c_{S}(a, \tilde{\mu})$ as a function of $a$. Those coefficients are positive in the region of $a$ considered here. Therefore, the bulk viscous correction effectively increases the Debye mass for $\Phi>0$.

In the absence of bulk viscous corrections, namely in the thermal equilibrium, the fluctuation-dissipation theorem (FDT) holds,

$$
\bar{D}_{S}(P)=\left(1+2 f_{0}\left(p_{0}\right)\right) \operatorname{sgn}\left(p_{0}\right)\left(\bar{D}_{R}-\bar{D}_{A}\right)=\frac{2 T}{p_{0}} \operatorname{sgn}\left(p_{0}\right)\left(\bar{D}_{R}-\bar{D}_{A}\right)+O\left(p_{0}\right),
$$

and this ensures that the two masses are equal in the absence of bulk viscous corrections. The FDT is violated in non-equilibrium, and $\widetilde{m}_{D, R}$ and $\widetilde{m}_{D, S}$ can be different. Thus, the difference quantifies the extent of violation of the FDT. In the current situation, in fact, a modified version of FDT holds [82],

$$
\bar{D}_{S}(P)=\frac{2 T \lambda}{p_{0}} \operatorname{sgn}\left(p_{0}\right)\left(\bar{D}_{R}-\bar{D}_{A}\right)+O\left(p_{0}\right),
$$




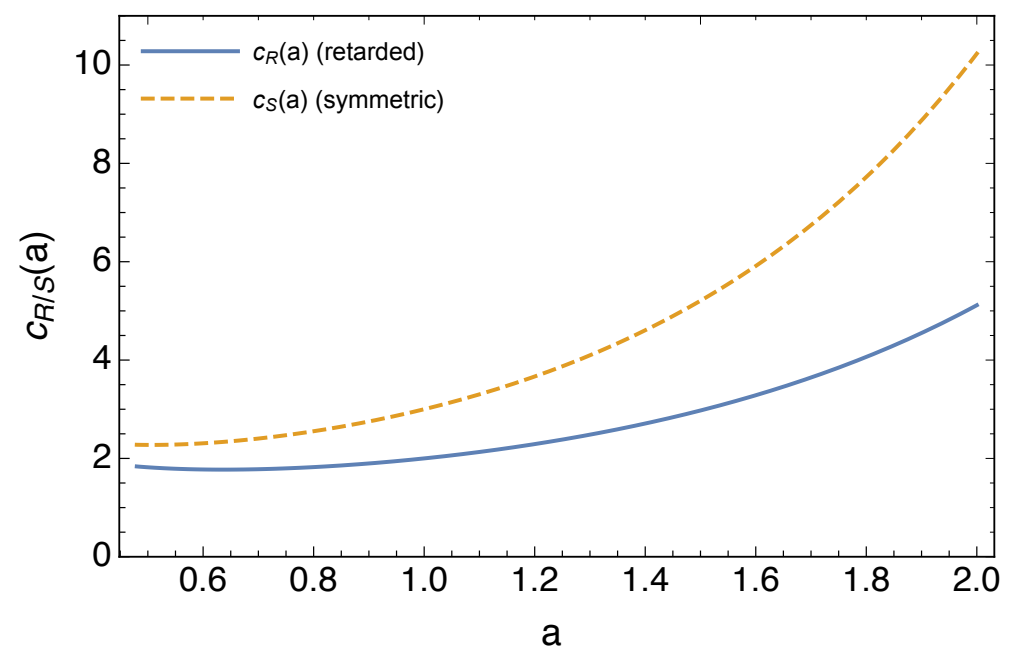

Figure 1. $a$-dependence of the functions $c_{S}(a)$ and $c_{R}(a)$, that are the slopes of $\widetilde{m}_{R(S)}^{2} / m_{D}^{2}$ as a function of $\Phi$. The chemical potential $\mu$ is set to zero.

or equivalently

$$
\Pi_{S}(P)=\frac{2 T \lambda}{p_{0}} \operatorname{sgn}\left(p_{0}\right)\left(\Pi_{R}-\Pi_{A}\right)+O\left(p_{0}\right),
$$

where we have defined a parameter

$$
\lambda=\lambda(a, \Phi, \tilde{\mu}) \equiv \frac{\widetilde{m}_{D, S}^{2}}{\widetilde{m}_{D, R}^{2}}=\frac{1+c_{S} \Phi}{1+c_{R} \Phi}
$$

The modified FDT (2.33) holds in the HTL approximation at one-loop and when the distribution function is spherically symmetric. As can be seen in figure 1, the symmetric Debye mass is larger than the retarded one for $\Phi>0$, so $\lambda>1$ in this case.

\section{In-medium potentials in the presence of bulk viscous corrections}

In this section, we study how the heavy quarkonia potential is modified in the presence of bulk viscous correction.

A heavy-quark potential can be obtained by the Fourier transform of the static gluon propagator. The propagators in the HTL perturbation theory results in the screened Coulombic potential. This potential will be dominant in the high temperature limit, but it does not account for the non-perturbative string-like part, which is responsible for the confinement. At zero or low temperature, many studies has confirmed that the so-called Cornell potential that consists of a Coulombic and string-like parts explain the properties of heavy quarkonia very well. Modeling of non-perturbative effect is important in understanding the "melting" of quarkonia near the crossover temperature $T_{c}$.

Given the medium property, how to incorporate it to modify the string-like contribution for both of the real and imaginary parts is not unique. There has been a number of proposals as to how to parametrize the in-medium potentials. Here, we shall discuss the prescriptions discussed in refs. [69, 79] and we extend those formulations to introduce the non-equilibrium bulk viscous corrections. We examine how the real and imaginary part of the in-medium potential is affected by this. 


\subsection{Approach based on the linear response}

We here take the approach [41] based on the linear response theory. The properties of a thermal medium is encoded in the dielectric permittivity $\varepsilon(p)$. When the linear approximation is justified, the in-medium potential is related to the potential in the vacuum through the permittivity by

$$
V(\mathbf{p})=V_{\text {vac }}(\mathbf{p}) \varepsilon^{-1}(\mathbf{p}) .
$$

This relation is true even in a strongly coupled system, as long as the linear approximation to the potential is good. As a vacuum potential, we employ the Cornell potential. Thus, the in-medium heavy-quark potential in the real space can be written as

$$
V(r)=\int \frac{d^{3} \mathbf{p}}{(2 \pi)^{3 / 2}}\left(e^{i \mathbf{p} \cdot \mathbf{r}}-1\right) \frac{V_{\text {Cornell }}(p)}{\varepsilon(p)},
$$

where the Fourier transform of the Cornell potential $V_{\text {Cornell }}(p)$ is given by

$$
V_{\text {Cornell }}(p)=-\sqrt{(2 / \pi)} \frac{\alpha}{p^{2}}-\frac{4 \sigma}{\sqrt{2 \pi} p^{4}},
$$

where $\alpha \equiv C_{F} \alpha_{s}$ with $C_{F}=\left(N_{c}^{2}-1\right) / 2 N_{c}$ and $\sigma$ is the string tension. The parameter $\sigma$ is determined to reproduce the vacuum quarkonium property.

Let us first look at the real part of the in-medium heavy quark potential. Using eqs. (2.30) and (3.3), it is computed as

$$
\begin{aligned}
\operatorname{Re} V(r) & =\int \frac{d^{3} \mathbf{p}}{(2 \pi)^{3 / 2}}\left(e^{i \mathbf{p} \cdot \mathbf{r}}-1\right) V_{\text {Cornell }}(p) \operatorname{Re} \varepsilon^{-1}(p) \\
& =-\alpha \widetilde{m}_{D, R}\left(\frac{e^{-\widetilde{m}_{D, R} r}}{\widetilde{m}_{D, R} r}+1\right)+\frac{2 \sigma}{\widetilde{m}_{D, R}}\left(\frac{e^{-\widetilde{m}_{D, R} r}-1}{\widetilde{m}_{D, R} r}+1\right) .
\end{aligned}
$$

where the first term is Coulombic contribution with the Debye screening, and the second term comes from the string-like part of the Cornell potential. In the absence of bulk viscous corrections, this form of potential is derived in ref. [41] . In the small distance limit, $r \rightarrow 0$, it approaches the Cornell potential, $V_{\text {Cornell }}(r)=-\alpha / r+\sigma r$. For the real part of the potential, the bulk viscous correction enters through the modification of the Debye mass $m_{D} \rightarrow \widetilde{m}_{D, R}$. In figure 2 , we plot the real part of the potential for different values of $\Phi$ (left) and $a$ (right). The modified Debye mass is an increasing function of both $\Phi$ and $a$, and the potential becomes flattened for larger values of those parameters.

The heavy quark potential also acquires an imaginary part at finite temperatures. The imaginary part reads

$$
\begin{aligned}
\operatorname{Im} V(r) & =\int \frac{d^{3} \mathbf{p}}{(2 \pi)^{3 / 2}}\left(e^{i \mathbf{p} \cdot \mathbf{r}}-1\right) V_{\text {Cornell }}(p) \operatorname{Im} \varepsilon^{-1}(p) \\
& =-\alpha \lambda T \phi_{2}\left(\widetilde{m}_{D, R} r\right)-\frac{2 \sigma T \lambda}{\widetilde{m}_{D, R}^{2}} \chi\left(\widetilde{m}_{D, R} r\right) \\
& \equiv \operatorname{Im} V_{\mathrm{HTL}}(r)+\operatorname{Im} V_{\text {string }}(r)
\end{aligned}
$$

where the first term is from the perturbative HTL contribution, and the second term is the string-like contribution. We have plotted these terms separately in figure 3 , and the 

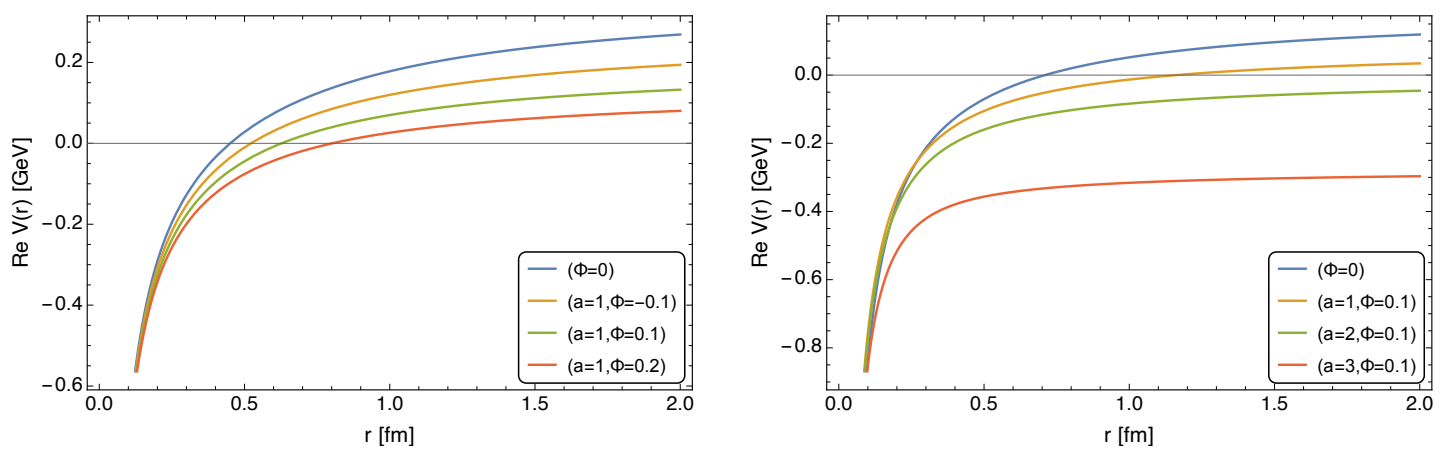

Figure 2. Left: real part of the potential $(\operatorname{Re} V)$ as a function of $r$ for different values of $\Phi$ with $a=1$ at $T=0.3 \mathrm{GeV}$. Right: $\operatorname{Re} V$ for different values of $a$ with $\Phi=0.1$ and $T=0.3 \mathrm{GeV}$. The potential becomes more flattened for larger values of $\Phi$ and $a$.
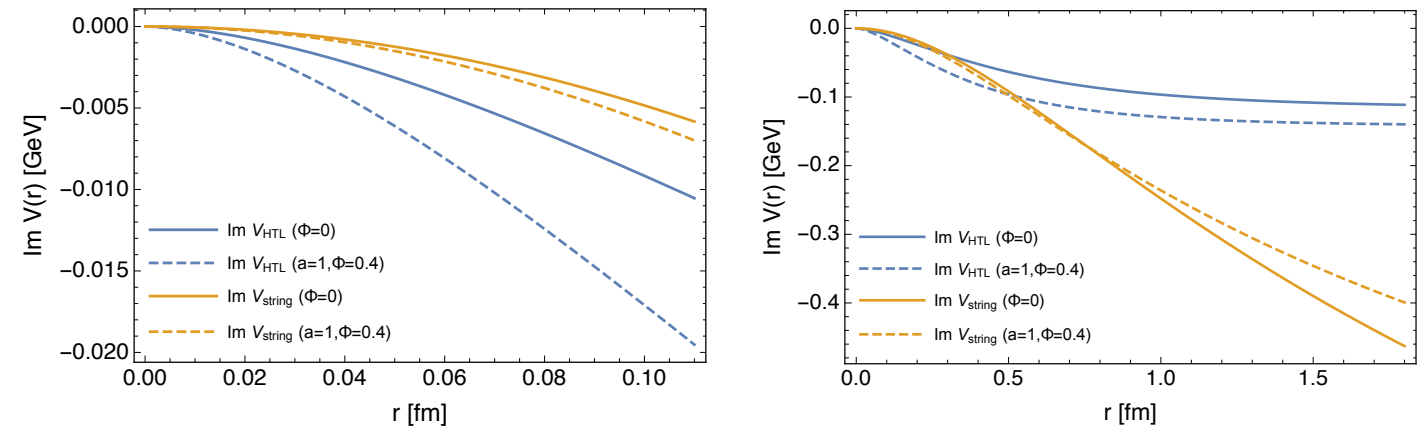

Figure 3. Imaginary part of the potentials with and without bulk viscous corrections at $T=$ $0.3 \mathrm{GeV}$. The contribution from the HTL part and the string-like part are shown separately. The left figure shows small $r$ region, and the right one shows a larger $r$ region.

total imaginary part in figure 4 . Note that the dimensionless parameter $\lambda$ is defined in eq. (2.35). The functions $\phi_{n}(x)$ and $\chi(x)$ are defined by

$$
\begin{aligned}
\phi_{n}(x) & \equiv 2 \int_{0}^{\infty} d z \frac{z}{\left(z^{2}+1\right)^{n}}\left[1-\frac{\sin (x z)}{x z}\right], \\
\chi(x) & \equiv 2 \int_{0}^{\infty} \frac{d z}{z\left(z^{2}+1\right)^{2}}\left[1-\frac{\sin (x z)}{x z}\right]
\end{aligned}
$$

The function $\phi_{2}(x)$ is a monotonically increasing function that asymptotes $\phi_{2}(0)=0$ and $\phi_{2}(\infty)=1 . \quad \chi(x)$ is also monotonically increasing with $\chi(0)=0$, but is logarithmically divergent at large $x .^{6}$

Let us examine the qualitative features of the imaginary part and its bulk viscous correction. We plot the imaginary part of the potential in figure 4. As can be seen in the figure, the bulk viscous correction on $\operatorname{Im} V$ is different in small $r$ and large $r$ regions. This can be understood as follows:

\footnotetext{
${ }^{6}$ In ref. [69], the physical origin of the divergence is identified to the absence of string breaking, and a way of regularization is discussed. On the value of the decay width that we later perform, this divergence is irrelevant because the wave function is localized.
} 


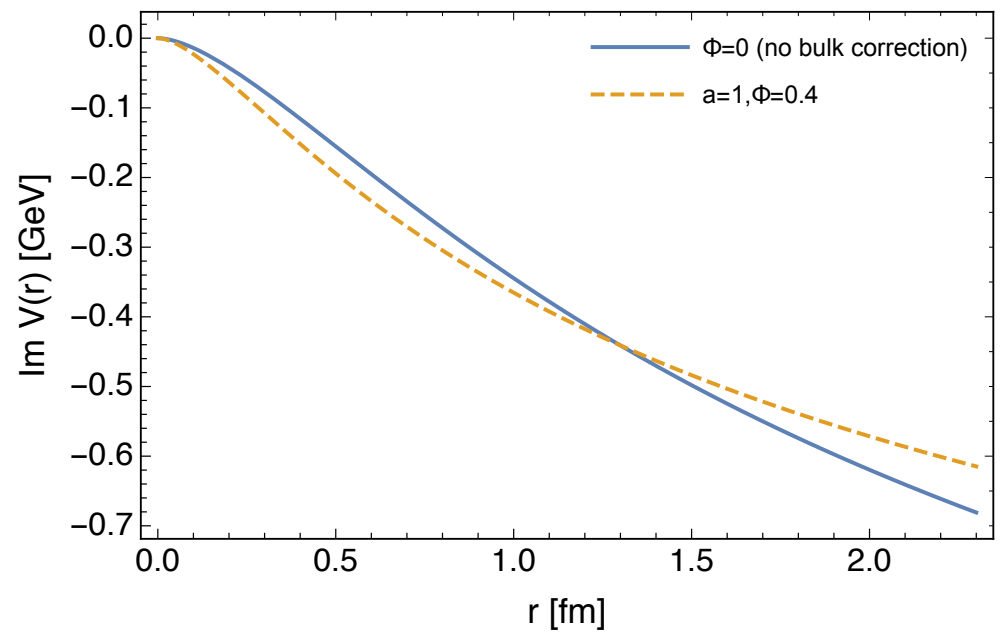

Figure 4. Imaginary part of the potential. $|\operatorname{Im} V|$ is enhanced in the small $r$ region, while it is suppressed at larger $r$.

- One consequence of the bulk viscous effect is a shift of the Debye mass,

$$
\frac{\widetilde{m}_{D, R}^{2}}{m_{D}^{2}}=1+c_{R}(a) \Phi
$$

The Debye mass becomes heavier due to the bulk correction for $\Phi>0$ and $\phi\left(\widetilde{m}_{D, R} r\right)$ increases with the bulk correction. The coefficient of the Coulombic term is $\alpha \lambda$, which is also an increasing function of $\Phi$. So, $\left|\operatorname{Im} V_{\mathrm{HTL}}\right|$ is an increasing function of $\Phi$. The perturbative HTL contribution is dominant in the small $r$ region, since $\phi_{2}\left(\widetilde{m}_{D, R} r\right) \sim r^{2} \ln r$ and $\chi \sim r^{2}$ to the leading order. See the left panel of figure 3 . Thus, in this region, $|\operatorname{Im} V|$ increases as $\Phi$ is increased.

- As shown in the right panel of figure 3, In the large $r$ region, string-like part dominates the imaginary part. In this region, $\left|\operatorname{Im} V_{\text {string }}\right|$ is suppressed in the presence of bulk correction with $\Phi>0$. The string part is proportional to the factor $\lambda / \widetilde{m}_{D, R}^{2}$, which decreases as a function of $\Phi$. Although $\chi\left(\widetilde{m}_{D, R} r\right)$ is an increasing function of $\Phi$, in total the string part decreases as a function of $\Phi$.

- As a result, for the bulk viscous correction for $\Phi>0$, we observe the enhancement of $|\operatorname{Im} V|$ in the small $r$ region, and suppression at large $r$, as shown in figure 4 .

\subsection{Introduction of non-perturbative propagator}

Let us discuss the prescription given in ref. [79]. In order to take into account the stringlike behavior of the potential, they have introduced a non-perturbative contribution to the resummed gluon propagator in addition to the HTL contribution as

$$
\bar{D}_{R}=\bar{D}_{R}^{\mathrm{p}}+\bar{D}_{R}^{\mathrm{np}},
$$

where $\bar{D}_{R}^{\mathrm{p}}=1 /\left(p^{2}-\Pi_{R}\right)$ is the perturbative HTL contribution. The non-perturbative contribution to the temporal component of the resummed gluon propagator is modeled by the following form,

$$
\bar{D}_{R}^{\mathrm{np}}(P)=b \frac{m_{G}^{2} m_{D}^{2}}{\left(p^{2}-\Pi_{R}\right)^{3}}+b^{\prime} \frac{m_{G}^{2}\left(-m_{D}^{2}-\Pi_{R}\right)}{\left(p^{2}-\Pi_{R}\right)^{3}},
$$


where $b=4$ and $b^{\prime}=6$ is chosen so that the leading contribution to the imaginary part of the potential in the small $r$ limit behaves as $r^{4} \ln r$. The mass scale $m_{G}$ is related to the string tension as $m_{G}^{2}=2 \sigma / \alpha$ by matching the small $r$ behavior of the real part of the potential with the Cornell potential. To get the symmetric propagator, the authors used the relation

$$
\bar{D}_{S}=\bar{D}_{R} \Pi_{S} \bar{D}_{A}=\frac{\Pi_{S}}{\Pi_{R}-\Pi_{A}}\left(\bar{D}_{R}-\bar{D}_{A}\right) .
$$

Using the equilibrium self-energies (2.5) and (2.19) in the HTL approximation, the nonperturbative part of the symmetric propagator in the static limit reads

$$
\bar{D}_{S}^{\mathrm{np}}\left(p_{0}=0, \mathbf{p}\right)=12 \pi i T m_{G}^{2} m_{D}^{2} \frac{p^{2}-m_{G}^{2}}{p\left(p^{2}+m_{D}^{2}\right)^{4}} .
$$

The Fourier transform of the propagators leads to the potential, 7,8

$$
\begin{aligned}
& \operatorname{Re} V_{\mathrm{GDPM}}(r)=-\alpha\left(\frac{e^{-m_{D} r}}{r}+m_{D}\right)+\frac{2 \sigma}{m_{D}}\left(1-e^{-m_{D} r}\right)-\sigma r e^{-m_{D} r}, \\
& \operatorname{Im} V_{\mathrm{GDPM}}(r)=-\alpha \phi_{2}\left(m_{D} r\right)+\frac{8 \sigma T}{m_{D}^{2}}\left[\phi_{3}\left(m_{D} r\right)-3 \phi_{4}\left(m_{D} r\right)\right] .
\end{aligned}
$$

Let us make a comment on the derivation. The use of eq. (3.11) might look problematic, because when we modify the resummed propagator as eq. (3.9), the self-energies should in general be modified and be different from the perturbative one. The Dyson-Schwinger equation (2.25) can be solved by the following expression,

$$
\bar{D}_{S}(P)=c \operatorname{sgn}\left(p_{0}\right)\left(\bar{D}_{R}-\bar{D}_{A}\right)-c \operatorname{sgn}\left(p_{0}\right) \bar{D}_{R}\left(\Pi_{R}-\Pi_{A}\right) \bar{D}_{A}+\bar{D}_{R} \Pi_{S} \bar{D}_{A}
$$

The parameter $c$ can be in fact arbitrary, because the sum of the first two terms on the right hand side is zero. In the thermal equilibrium, the most convenient choice is $c=1+2 f_{0}$, where $f_{0}=f_{0}\left(p_{0}\right)$ is the Bose distribution. It is convenient because the FDT holds in the thermal equilibrium,

$$
\Pi_{S}(P)=\left(1+2 f_{0}\right) \operatorname{sgn}\left(p_{0}\right)\left(\Pi_{R}-\Pi_{A}\right),
$$

because of which the last two terms of eq. (3.15) are equal in magnitude. Therefore, in equilibrium, the symmetric propagator can be expressed in three equivalent ways,

$$
\begin{aligned}
\bar{D}_{S}(P) & =\left(1+2 f_{0}\right) \operatorname{sgn}\left(p_{0}\right)\left(\bar{D}_{R}-\bar{D}_{A}\right) \\
& =\left(1+2 f_{0}\right) \operatorname{sgn}\left(p_{0}\right) \bar{D}_{R}\left(\Pi_{R}-\Pi_{A}\right) \bar{D}_{A} \\
& =\bar{D}_{R} \Pi_{S} \bar{D}_{A} .
\end{aligned}
$$

If we use the first expression, it is evident that when $\bar{D}_{R}$ has an additive contribution as eq. (3.9), the symmetric propagator gets an additive contribution as $\bar{D}_{S}=\bar{D}_{S}^{\mathrm{p}}+\bar{D}_{S}^{\mathrm{np}}$. Namely, eq. (3.11) does not actually depend on the self-energies.

\footnotetext{
${ }^{7}$ The one given here is the second model discussed in ref. [79]. In their first model, the non-perturbative retarded propagator is modeled as

$$
\bar{D}_{R}^{\mathrm{np}}(P)=\frac{m_{G}^{2}}{\left(p^{2}-\Pi_{R}\right)^{2}} .
$$

The real part of this propagator is $m_{G}^{2} /\left(p^{2}+m_{D}^{2}\right)^{2}$ and is the same as the non-perturbative propagator discussed in ref. [83]. This choice results in the KMS potential [13] for the real part.

${ }^{8}$ The same real part of the potential is obtained in ref. [50] through a different line of reasoning.
} 
From this consideration, it might seem difficult to extend this prescription to nonequilibrium. This is because, we do not have the FDT in non-equilibrium, and we have to use eq. (3.11) to compute the resummed symmetric propagator, but there is no way to determine $\Pi_{S}$ when there are perturbative and non-perturbative contributions in $\bar{D}_{R}$. However, in the current case of the bulk viscous correction, we can determine the $\bar{D}_{S}$ even though it is non-equilibrium, by assuming a modified FDT (2.34). ${ }^{9}$ We can choose the parameter as $c=2 T \lambda / p_{0}$ and we can express $\bar{D}_{S}$ as

$$
\bar{D}_{S}(P)=\frac{2 T \lambda}{p_{0}} \operatorname{sgn}\left(p_{0}\right)\left(\bar{D}_{R}-\bar{D}_{A}\right)-\frac{2 T \lambda}{p_{0}} \operatorname{sgn}\left(p_{0}\right) \bar{D}_{R}\left(\Pi_{R}-\Pi_{A}\right) \bar{D}_{A}+\bar{D}_{R} \Pi_{S} \bar{D}_{A} .
$$

Because of the modified FDT (2.34), and the last two terms in eq. (3.18) cancel, just like the case of equilibrium. Therefore, we can express the resummed symmetric propagator in three ways,

$$
\begin{aligned}
\bar{D}_{S}(P) & =\frac{2 T \lambda}{p_{0}} \operatorname{sgn}\left(p_{0}\right)\left(\bar{D}_{R}-\bar{D}_{A}\right) \\
& =\frac{2 T \lambda}{p_{0}} \operatorname{sgn}\left(p_{0}\right) \bar{D}_{R}\left(\Pi_{R}-\Pi_{A}\right) \bar{D}_{A} \\
& =\bar{D}_{R} \Pi_{S} \bar{D}_{A} .
\end{aligned}
$$

In the first expression, it is evident that when the retarded (or advanced) propagator gets an additive contribution as eq. (3.9), the non-perturbative contribution additively contribute to $\bar{D}_{S}=\bar{D}_{S}^{\mathrm{p}}+\bar{D}_{S}^{\mathrm{np}}$.

In the presence of bulk viscous corrections, let us we consider the same form of the non-perturbative contribution to the retarded self-energy,

$$
\bar{D}_{R}^{\mathrm{np}}(P)=b \frac{m_{G}^{2} \widetilde{m}_{D, R}^{2}}{\left(p^{2}-\Pi_{R}\right)^{3}}+b^{\prime} \frac{m_{G}^{2}\left(-\widetilde{m}_{D, R}^{2}-\Pi_{R}\right)}{\left(p^{2}-\Pi_{R}\right)^{3}},
$$

where $\Pi_{R}$ is given by eq. (2.9). Note that the second term does not contribute to the real part of the potential, because its real part vanishes in the static limit $p_{0} \rightarrow 0$. We can compute the corresponding non-perturbative part of the resummed symmetric propagator using eq. (3.19) as

$$
\bar{D}_{S}^{\mathrm{np}}\left(p_{0}=0, \mathbf{p}\right)=12 \pi i T \lambda m_{G}^{2} \widetilde{m}_{D, R}^{2} \frac{p^{2}-m_{G}^{2}}{p\left(p^{2}+\widetilde{m}_{D, R}^{2}\right)^{4}} .
$$

The difference from the equilibrium case (3.12) is the replacement $m_{D} \rightarrow \widetilde{m}_{D, R}$ and the multiplication by $\lambda$.

Now that we got the expression of the necessary propagators and we are ready to compute the potential through eq. (2.29). The real part can be obtained by a simple replacement of the Debye mass $m_{D} \rightarrow \widetilde{m}_{D, R}$. Using the modified symmetric propagator (3.21), the imaginary part of the potential is given by

$$
\operatorname{Im} V_{\mathrm{GDPM}}^{\mathrm{bulk}}(r)=-\alpha \lambda \phi_{2}\left(\widetilde{m}_{D, R} r\right)+\frac{8 \sigma T \lambda}{\widetilde{m}_{D, R}^{2}}\left[\phi_{3}\left(\widetilde{m}_{D, R} r\right)-3 \phi_{4}\left(\widetilde{m}_{D, R} r\right)\right] .
$$

\footnotetext{
${ }^{9}$ The modified FDT is derived in the perturbation theory and whether it is still valid in the nonperturbative regime can be questioned.
} 

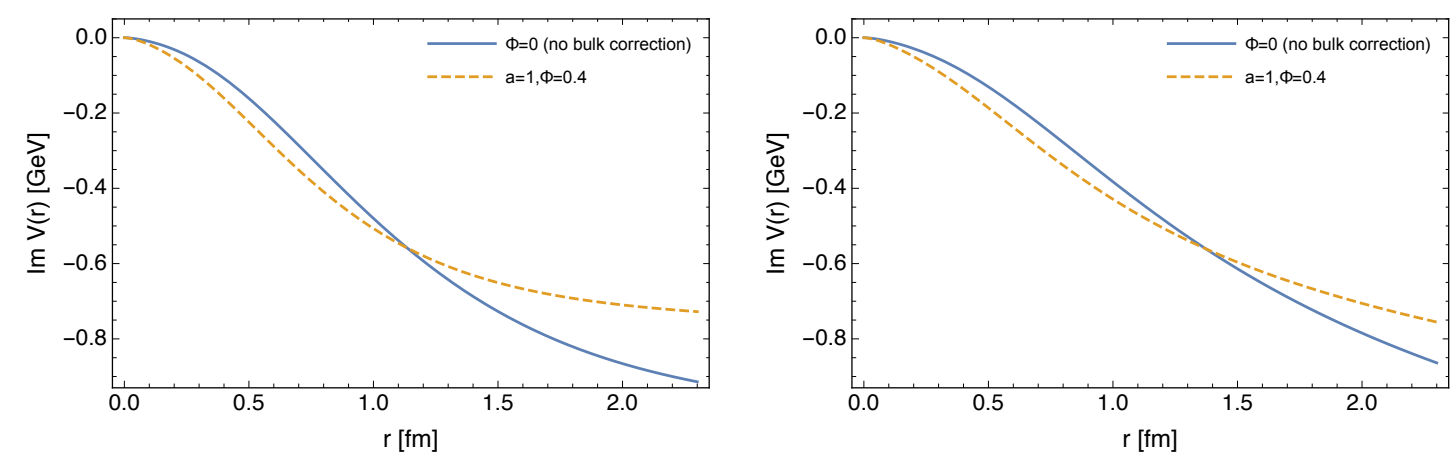

Figure 5. Imaginary part of the potential obtained via the prescription of ref. [79] (left) and ref. [69] (right). The potentials without bulk viscous correction are denoted by solid lines, and dashed lines are with the corrections. In both prescriptions, $|\operatorname{Im} V|$ is enhanced in the small $r$ region, while it is suppressed at larger $r$. The parameters are set as $T=0.3 \mathrm{GeV}, \sigma=(0.44)^{2} \mathrm{GeV}^{2}$, and $\alpha=0.392$.

On the left panel of figure 5, we plot the imaginary part of the potential for both equilibrium and non-equilibrium cases. Qualitatively, how the imaginary part is affected is the same as the case (3.5). $|\operatorname{Im} V|$ gets enhanced at small $r$, and suppressed at large $r$.

\subsection{Approach based on a generalized Gauss law}

In ref. [69], a different prescription to obtain the potential is given. They have used a generalized Gauss law, that can be applicable to a string-like potential, and the HTL permittivity to obtain an analytic form of the in-medium potential. The real part of the potential is the same as eq. (3.13). The imaginary part of the potential in the thermal equilibrium is given by

$$
\operatorname{Im} V_{\mathrm{LR}}(r)=-\alpha T \phi_{2}\left(m_{D} r\right)-\frac{\sqrt{\pi}}{4} T \sigma m_{D} r^{3} G_{2,4}^{2,2}\left(\begin{array}{c|c}
-\frac{1}{2},-\frac{1}{2} & \frac{m_{D}^{2} r^{2}}{4}
\end{array}\right),
$$

where $G$ is Meijer's $G$-function. We can use the same prescription straightforwardly to obtain the potential in the presence of the bulk viscous corrections, by using the modified dielectric permittivity (2.30). The real part is again just a replacement $m_{D} \rightarrow \widetilde{m}_{D, R}$. The imaginary part is given by

$$
\operatorname{Im} V_{\mathrm{LR}}^{\text {bulk }}(r)=-\alpha \lambda T \phi_{2}\left(\widetilde{m}_{D, R} r\right)-\frac{\sqrt{\pi}}{4} T \sigma \lambda \widetilde{m}_{D, R} r^{3} G_{2,4}^{2,2}\left(\begin{array}{c|c}
-\frac{1}{2},-\frac{1}{2} & \widetilde{m}_{D, R}^{2} r^{2} \\
\frac{1}{2}, \frac{1}{2},-\frac{3}{2},-1
\end{array}\right) .
$$

In this prescription too, the way the bulk correction affects the potential is also very similar to the case (3.5). At small $r$, the second term is $r^{4}$ while the first term is $r^{2} \ln r$, so the first term is dominant, where its magnitude is enhanced for $\Phi>0$. At large $r$, the second term is dominant. On the right panel of figure 5, we plot the imaginary part (3.24) with and without bulk corrections. For $\Phi>0,\left|\operatorname{Im} V_{\mathrm{LR}}\right|$ is enhanced in the small $r$ region, and suppressed at large $r$.

\subsection{Comparison of the real part of the potentials}

Let us conclude this section by giving a comparison of several models of heavy quark potentials in the thermal equilibrium. In figure 6, we plot the potentials (3.4) (denoted 


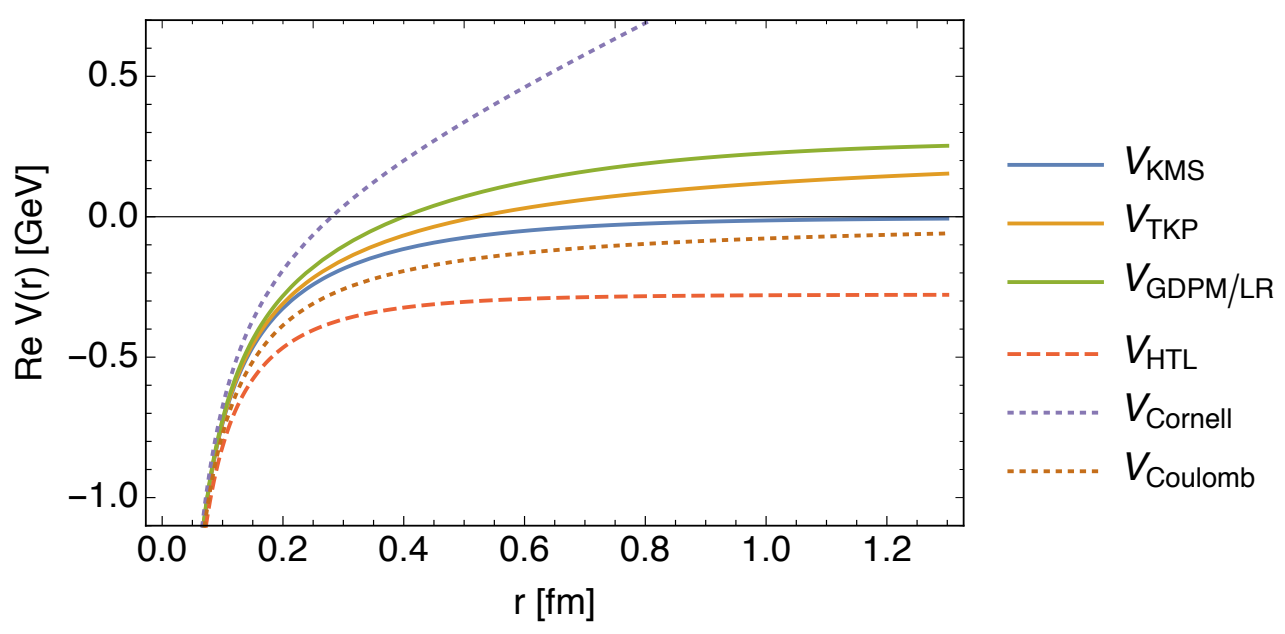

Figure 6. Comparison of the models of the real part of the in-medium potential.

by $\left.V_{\mathrm{TKP}}\right)$ and (3.13) (denoted by $V_{\mathrm{GDPM} / \mathrm{LR}}$ ) in the absence of bulk viscous corrections. We have also plotted one of the previous model of the finite temperature potential, given by [13]

$$
V_{\mathrm{KMS}}(r)=-\frac{\alpha}{r} e^{-m_{D} r}+\frac{\sigma}{m_{D}}\left(1-e^{-m_{D} r}\right) .
$$

All the models are parametrized by the Debye mass $m_{D}$, strong coupling constant $\alpha$, and the string tension $\sigma$. We take common values for the plot. Namely, all the models asymptote to the Cornell potential with the same parameters in the small distance limit. For a comparison, we also plotted the perturbative HTL potential (the first term of eq. (3.4)), the Cornell potential, and Coulomb potential. The potentials Re $V_{\mathrm{KMS}}, \operatorname{Re} V_{\mathrm{TKP}}$, and $\operatorname{Re} V_{\mathrm{GDPM} / \mathrm{LR}}$ start to deviate from each other around the length scale given by the inverse Debye mass.

\section{Heavy quarkonia in the presence of bulk viscous corrections}

In this section, we discuss the effect of bulk corrections on the properties of heavy quarkonia, such as the binding energies and decay widths, based on the potential obtained in the section 3.1.

\subsection{Computational setup}

Let us describe the computational procedure. In order to study the in-medium properties of quarkonia, we here solve the Schrödinger equation for a heavy quarkonium to obtain the wave function, using the real part of the in-medium potential.

The potential is the function of only the radial coordinate and we only have to solve the ordinary differential equation of the radial part of the wave function. The time-independent Schrödinger equation for the radial wave function reads

$$
-\frac{1}{2 m_{q}}\left(\psi^{\prime \prime}(r)+\frac{2}{r} \psi^{\prime}(r)-\frac{\ell(\ell+1)}{r^{2}} \psi(r)\right)+\operatorname{Re} V(r) \psi(r)=\epsilon_{n \ell} \psi(r),
$$


where $m_{q}$ is the reduced mass of the quarkonium system. We numerically solve this using the real part of the potential (3.4) modified by the bulk viscous correction to obtain the wave functions and eigenvalues. ${ }^{10}$

The binding energy is given by the difference between the asymptotic value of the potential and the eigenvalue $\epsilon_{n \ell}$,

$$
E_{\text {bin }}=\operatorname{Re} V(r \rightarrow \infty)-\epsilon_{n \ell},
$$

In the case of the potential (3.4), the asymptotic value is

$$
\operatorname{Re} V(r \rightarrow \infty)=-\alpha \widetilde{m}_{D, R}+\frac{2 \sigma}{\widetilde{m}_{D, R}}
$$

Using the imaginary part of the potential (3.5), we can make an estimate for the thermal decay width $\Gamma$ by

$$
\Gamma=-\langle\psi|\operatorname{Im} V(r)| \psi\rangle=-\frac{\int d r r^{2}|\psi(r)|^{2} \operatorname{Im} V(r)}{\int d r r^{2}|\psi(r)|^{2}} .
$$

In a thermal environment, the wave function cannot exist as a steady state, but is transient. This way of the estimate of the decay width treat the imaginary part of the potential as a perturbation and its validity worsens when the decay width become comparable the binding energy.

Below is how the parameters are set:

- For the Debye masses, we use the perturbative expressions (2.10), (2.21) for different temperatures/chemical potentials, including the bulk viscous corrections. Since potentials are parametrized by the Debye mass one could regard the Debye masses as a parameter and fit it to reproduce the potential computed from the lattice QCD data. We do not take this approach here and use the HTL expression, since the primary focus in this study is to understand the nature of bulk viscous modification on heavy-quark properties.

- We set the reduced heavy quark mass to $m_{q}=1.25 / 2 \mathrm{GeV}$ for $c \bar{c}$ and $m_{q}=4.66 / 2 \mathrm{GeV}$ for $b \bar{b}$.

- The string tension is chosen to $\sigma=(0.44 \mathrm{GeV})^{2}$.

- For the coupling constant, we use the one-loop result,

$$
\alpha_{s}=\frac{g^{2}}{4 \pi}=\frac{6 \pi}{\left(11 N_{c}-2 N_{f}\right) \log \left(2 \pi \sqrt{T^{2}+\mu^{2} / \pi^{2}} / \Lambda\right)},
$$

\footnotetext{
${ }^{10}$ The interpretation of the role of a complex potential needs some care. As shown in [33], the complex potential dictates the time evolution of unequal time point-split meson-meson correlator, which is related to spectral functions, and the binding energies and decay widths can be read off from the spectral functions. Strictly speaking, the potential does not describe the time evolution of the wave function itself. In this study, we used the real part to dictate the wave function itself and computed the binding energies and decay widths based on the wave function. An advantage of this approach is that it gives us an intuitive picture on the behavior of heavy quarkonia in the medium. The resultant melting temperature is in agreement with those obtained from the approach [33]. When the decay width becomes comparable to the binding energy, it would be better to directly compute the spectral function.
} 

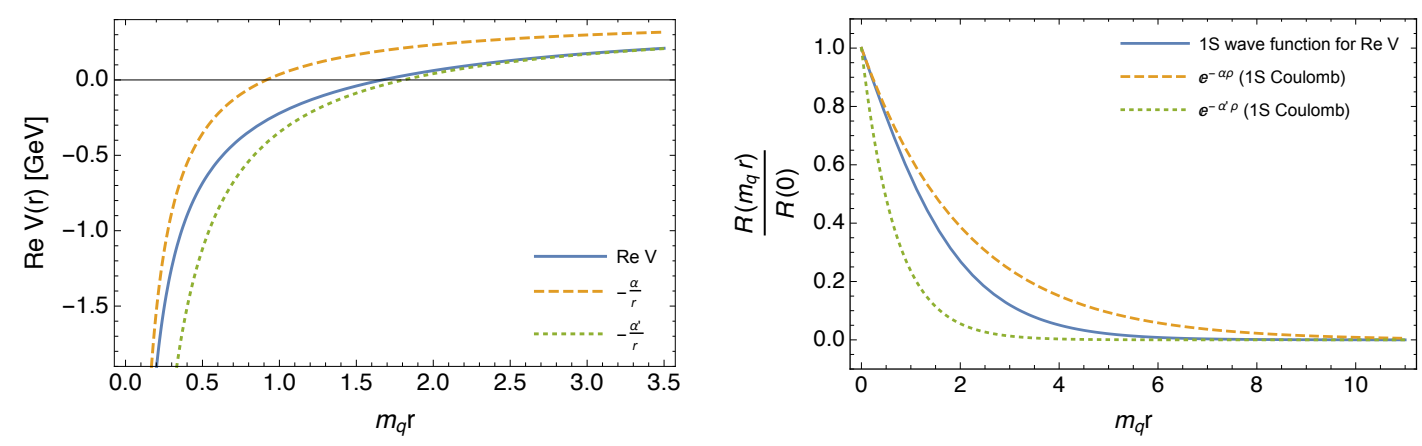

Figure 7. Left: real part of the in-medium potential (3.4) at $T=0.3 \mathrm{GeV}, \mu=0$ and $\Phi=0$ in comparison with its asymptotic Coulomb potentials. Right: radial wave function $R\left(m_{q} r\right)$ of $J / \psi$ state. The ground states of the asymptotic Coulomb potentials are shown for comparison.

with $N_{c}=N_{f}=3$. Namely the renormalization scale is taken to be $2 \pi \sqrt{T^{2}+\mu^{2} / \pi^{2}}$. The scale $\Lambda$ is chosen to $\Lambda=0.176 \mathrm{GeV}$ requiring $\alpha_{S}(1.5 \mathrm{GeV})=0.326$ is satisfied to match the lattice measurements [84].

Under the current setting, we expect out computation becomes less trustable at lower temperatures. The Debye mass would deviate from $\propto T$ behavior, and the value of the coupling would deviate from the 1-loop results. This point may be improved by extracting the Debye mass and the coupling from lattice QCD results.

\subsection{Wave function}

Let us first discuss the qualitative feature of the wave functions of quarkonia from the finite temperature potential (3.4). At $r \rightarrow 0$, the potential approaches a Coulomb potential with coefficient $\alpha$,

$$
\operatorname{Re} V \sim-\frac{\alpha}{r}
$$

On the other hand, at large distances, the potential also approaches a Coulomb potential, but with a different coefficient,

$$
\operatorname{Re} V \sim-\frac{\alpha^{\prime}}{r}
$$

with $\alpha^{\prime} \equiv 2 \sigma / \widetilde{m}_{D, R}^{2}$. The switching of those two regimes happens around $r$ given by the inverse Debye mass. In the limit of the large Debye mass (or high temperature), this part is flattened since the coefficient $\alpha^{\prime}$ goes to zero, and the potential is dominated by the screened HTL one. In the left panel of figure 7, we plot the potential as well as its asymptotic Coulomb potentials. In the right panel of figure 7 , we show the ground state wave function from the potential (3.4) normalized at $r=0$, as well as those from the asymptotic Coulomb potentials, at $T=0.3 \mathrm{GeV}$. At this temperature, the Debye mass $\sim 0.63 \mathrm{GeV}$ is comparable to the reduced mass of the charm quark, and the wave function is away from both of the two Coulomb wave functions.

In figure 8, we show how the wave function is deformed as the temperature goes higher, for the ground state and the first excited states with $\ell=0$. A larger temperature results in a larger Debye mass, because of which the wave function is more delocalized and the size of the quarkonium becomes larger. Since the bulk viscous correction comes through 

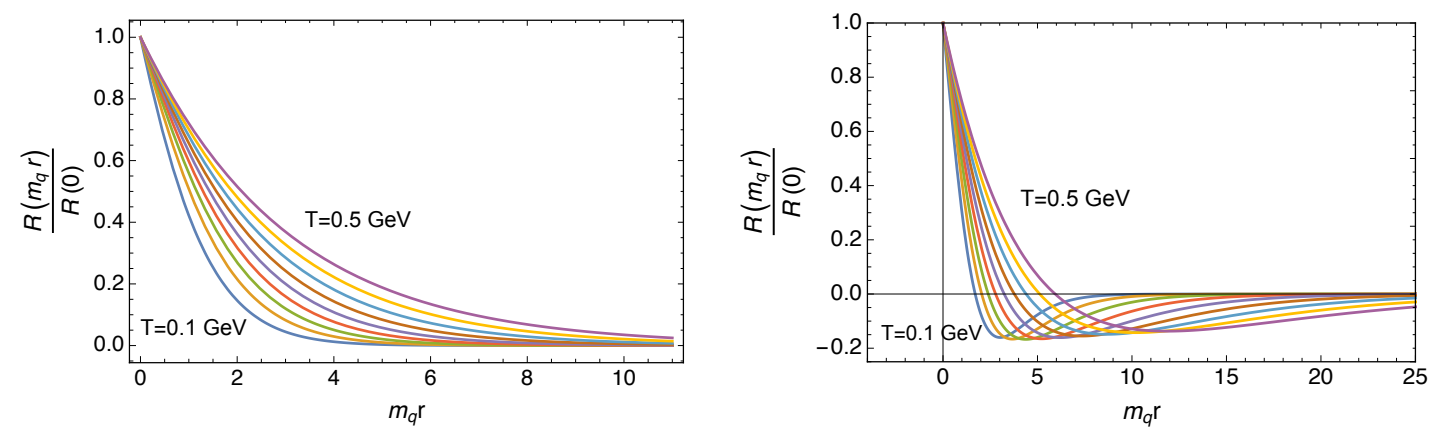

Figure 8. Radial wave functions of the ground state (left) and first excited state (right) of $c \bar{c}$ wave functions at different temperatures, as a function of $m_{q} r$. The temperature is changed from $0.1 \mathrm{GeV}$ to $0.5 \mathrm{GeV}$ by $0.05 \mathrm{GeV}$.

the modification of the Debye mass, for the real part of the potential. When $\Phi>0$, the Debye mass becomes larger, and the wave function becomes more delocalized.

\subsection{Binding energies and decay widths}

\subsubsection{Dependence on the scale $\Lambda$}

Let us first consider the case without bulk viscous corrections. Figure 9 shows the binding energies and decay widths for $J / \psi, \Upsilon$, and $\Upsilon^{\prime}$ states. As a check of the systematic dependence, we have changed the value of the scale $\Lambda$ by factors of $1 / 2$ to 2 , and different lines correspond to different values of $\Lambda$. For $J / \psi$ and $\Upsilon^{\prime}$, the binding energies decrease and the decay widths increase for a larger $\Lambda$. $\Upsilon$ shows a different behavior: the scale dependence changes around $T=0.25 \mathrm{GeV}$.

The difference in behavior is related to the size of the wave function. In figure 10, we show the mean radius of the quarkonia states, $\bar{r} \equiv \sqrt{\left\langle r^{2}\right\rangle}$, with

$$
\left\langle r^{2}\right\rangle=\frac{\int d r r^{2}|\psi(r)|^{2} r^{2}}{\int d r r^{2}|\psi(r)|^{2}}
$$

At around $T=0.25 \mathrm{GeV}$, the mean radius of $\Upsilon$ becomes larger than the inverse Debye mass. When the wave function is small compared to the inverse Debye mass, the wave function is not sensitive to the screening and its behavior is determined by the Cornell-like potential. In the limit of tight wave function (large mass), the shape of the wave function is qualitatively close to the Coulomb wave function. Then, the binding energy is given by

$$
E_{\text {bin }} \simeq \frac{m_{q} \alpha^{2}}{2} \text {. }
$$

Since $\alpha$ is an increasing function of $\Lambda$, the binding energy increases for larger $\Lambda, \frac{\partial \alpha}{\partial \Lambda}>0$. On the other hand, at higher temperature, the size of the wave function grows and the Debye mass becomes relevant. The potential asymptotically approaches a Coulomb potential with a different coefficient $\alpha^{\prime} \equiv 2 \sigma / \widetilde{m}_{D, R}^{2}$. In this situation, the binding energy reads

$$
E_{\mathrm{bin}} \simeq \frac{m_{q}\left(\alpha^{\prime}\right)^{2}}{2}=\frac{2 m_{q} \sigma^{2}}{m_{D}^{4}} \propto \frac{1}{\alpha^{2}}
$$



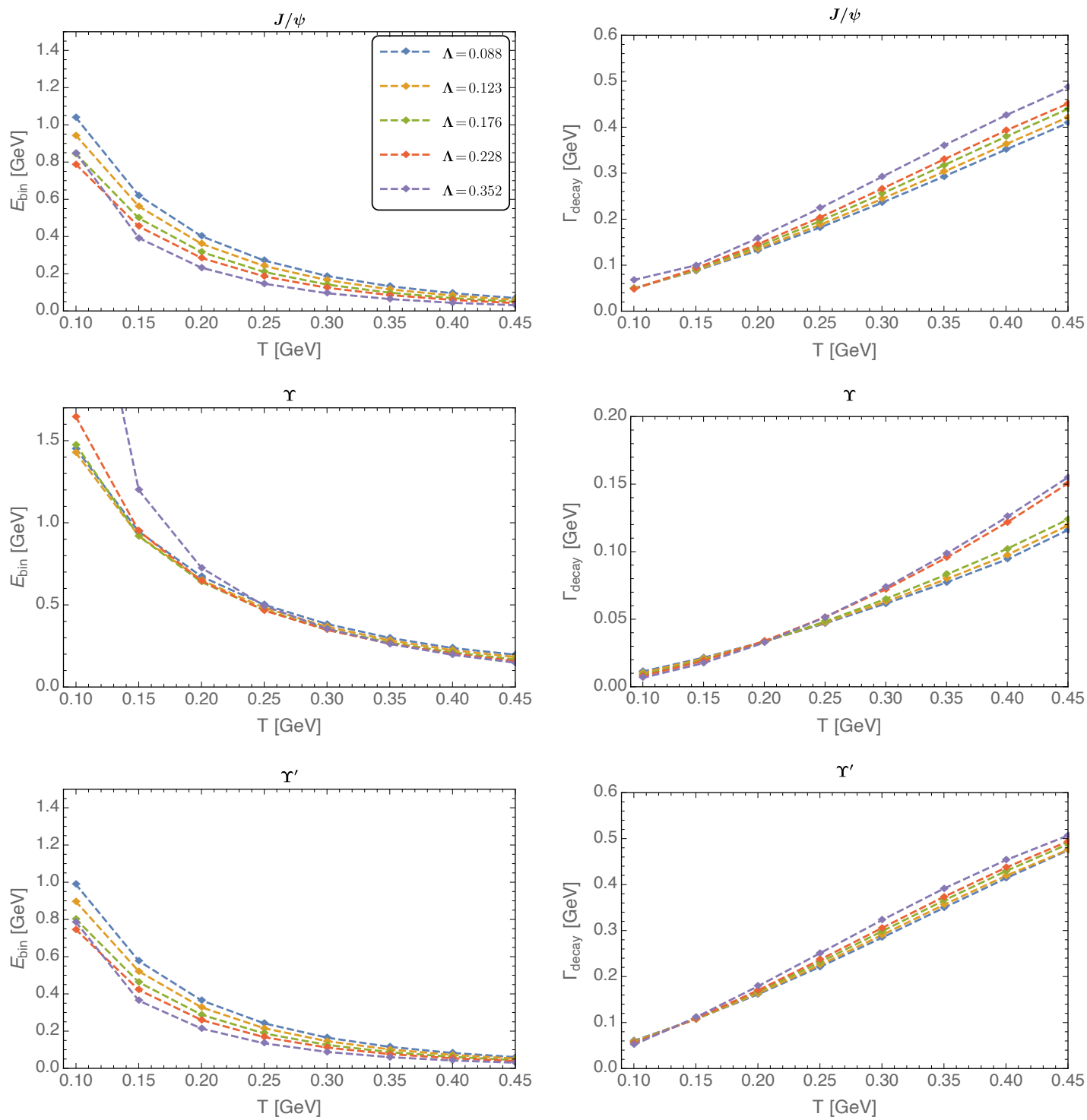

Figure 9. Binding energies (left column) and decay widths (right column) for $J / \psi$ (top), $\Upsilon$ (middle), and $\Upsilon^{\prime}$ (bottom) as a function of temperature. Different colors correspond to different values of the scale $\Lambda$.

Therefore, when the size of the wave function is comparable or larger than the Debye screening length, the binding energy is a decreasing function of $\Lambda$. This is why the $\Lambda$ dependence of the binding energy changes around $T=0.25 \mathrm{GeV}$ for $\Upsilon$. In the case of $J / \psi$ and $\Upsilon^{\prime}$, the mean radius is larger than the inverse Debye mass, and the binding energy is always an increasing function of the scale $\Lambda$.

\subsubsection{Effect of bulk viscous corrections}

Now let us discuss the effect of the bulk viscous corrections on the binding energies and decay widths. In figure 11, we present the binding energies and decay widths for charmonium (top) and bottomonium (bottom) states as a function of temperature. The solid lines correspond to the cases without bulk correction, and the dotted lines are with the bulk 


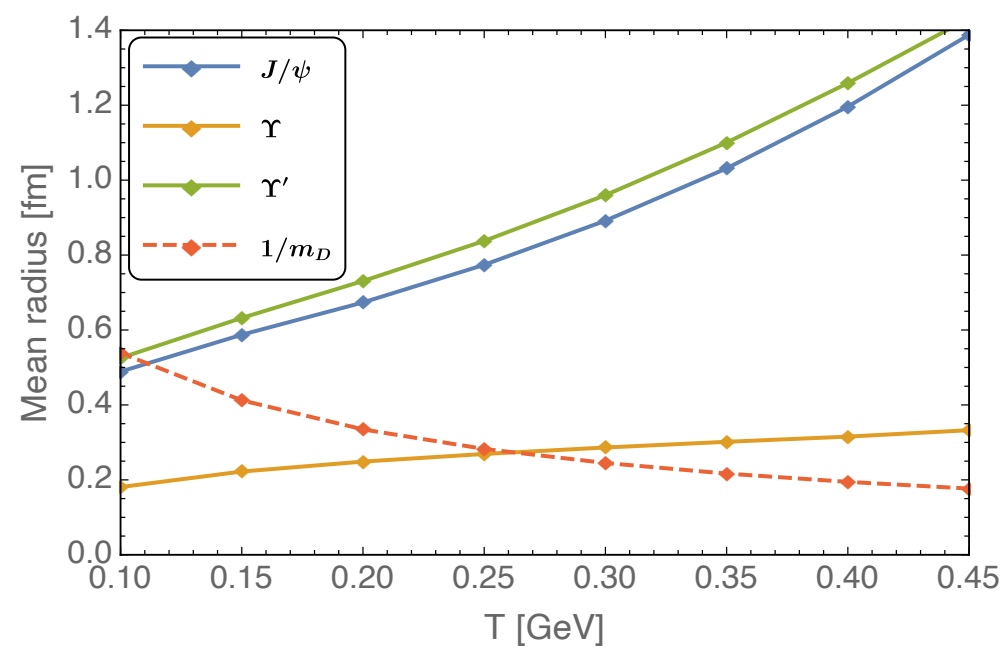

Figure 10. Mean radius of $J / \psi, \Upsilon$, and $\Upsilon^{\prime}$ compared with the Debye screening length $1 / m_{D}$, as a function of temperature.
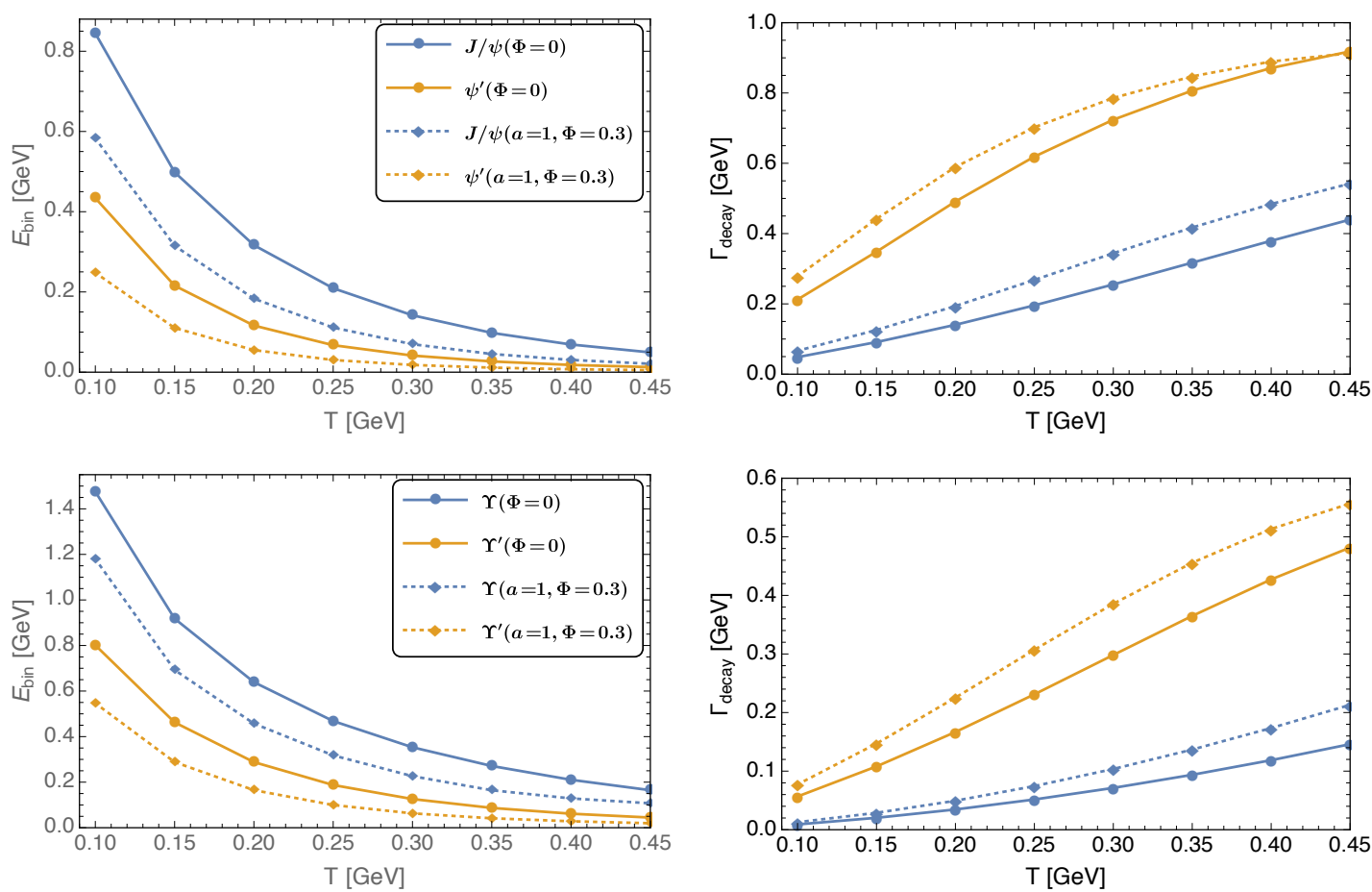

Figure 11. Modification of the binding energies (left) and decay widths (right) for charmonium (top) and bottomonium (bottom) states due to the bulk viscous corrections. Solid lines are those without the bulk corrections and dotted lines are with the modified ones.

correction. Generically, the bulk correction for $\Phi>0$ lowers the binding energies, since the bulk viscous correction make the Debye mass heavier and the screening becomes stronger. For a negative $\Phi$, the effect is opposite.

As for the decay widths, as shown in the right column of figure 11, the magnitude of the decay width is enhanced in the presence of bulk viscous corrections. The decay width 


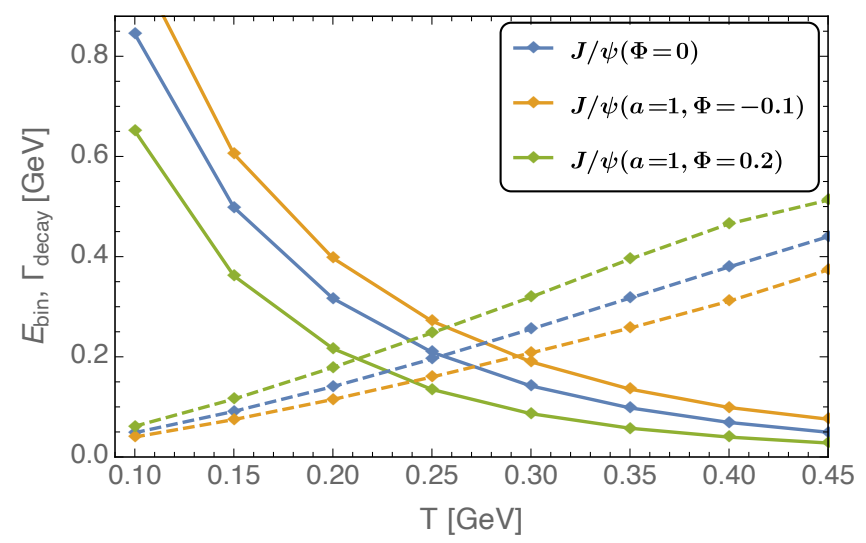

Figure 12. Shift of the melting temperature. The temperature dependence of the binding energies (solid lines) and decay widths (dashed lines) of $J / \psi$ for different parameters $\Phi$ (indicated by different colors) is shown. The position of the intersection of a solid and dashed lines of the same color indicate the melting temperature for the corresponding parameter $\Phi$.
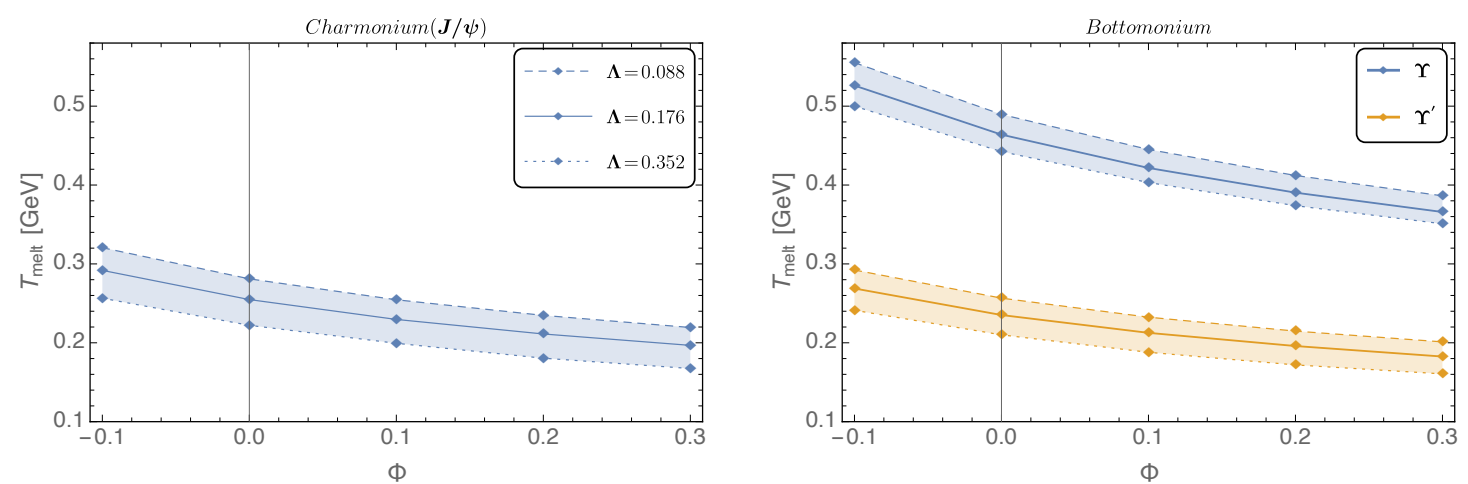

Figure 13. Melting temperature of $J / \psi$ (left), $\Upsilon$ and $\Upsilon^{\prime}$ (right) as a function of the bulk viscous parameter $\Phi$ with $a=1$. The solid lines corresponds to $\Lambda=0.176 \mathrm{GeV}$, around which the value of $\Lambda$ is varied by a factor of $0.5(\Lambda=0.5 \times 0.176 \mathrm{GeV}$, dashed line $)$, and a factor of $2(\Lambda=2 \times 0.176 \mathrm{GeV}$, dotted line).

of the $\psi^{\prime}$ state with the bulk correction (top-right) shows flattening at higher temperatures around $0.4 \mathrm{GeV}$. This is related to the nature of the imaginary part discussed in section 3.1. As we discussed there, in the presence of the bulk viscous correction, $|\operatorname{Im} V|$ is enhanced at small $r$, while it is suppressed at higher $r$. The wave function of $J / \psi$ is smaller in size, and in this region $|\operatorname{Im} V|$ is increased, resulting in the larger decay width. On the other hand, the excited states are larger and are more sensitive to the large $r$ part of $|\operatorname{Im} V|$. The enhancement of the decay width of $\psi^{\prime}$ become saturated, because the wave function is now in the region where $|\operatorname{Im} V|$ is suppressed by the bulk correction.

Based on the computations of the binding energies and decay widths, we can make an estimate of the melting temperature $T_{\text {melt }}$ of quarkonium states. We adopt a common criterion that the binding energy coincide with the decay width, $E_{\text {bin }}\left(T_{\text {melt }}\right)=\Gamma\left(T_{\text {melt }}\right)$. For example, in figure 12 , we plot the $E_{\text {bin }}$ (solid line) and $\Gamma$ (dashed line) of $J / \psi$ states. Different color corresponds to different parameter $\Phi$. The position at which a solid and dashed lines of the same color intersect is the melting temperature for the parameter $\Phi$. 


\begin{tabular}{|ccc|}
\hline & $T_{\text {melt }}[\mathrm{GeV}]$ & Ref. $[69]$ \\
\hline$J / \psi$ & $0.254_{-0.032}^{+0.027}$ & $0.267_{-0.036}^{+0.033}$ \\
$\Upsilon$ & $0.464_{-0.022}^{+0.026}$ & $0.440_{-0.055}^{+0.080}$ \\
$\Upsilon^{\prime}$ & $0.235_{-0.022}^{+0.025}$ & $0.250_{-0.053}^{+0.050}$ \\
\hline
\end{tabular}

Table 1. Melting temperatures for different states in the absence of bulk viscous correction $(\Phi=0)$ , compared with a recent work based on the lattice QCD [69].

In table 1, we compare the melting temperatures computed in the current setup (without bulk correction) as well as the results from ref. [69], that are based on the extraction of the potential with lattice QCD data and subsequent computation of spectral functions. In order to gain a sense of uncertainty, we varied the scale $\Lambda$ around $0.176 \mathrm{GeV}$, and the upper and lower numbers for our data in the table correspond to $\Lambda=1 / 2 \times 0.176 \mathrm{GeV}$ and $\Lambda=2 \times 0.176 \mathrm{GeV}$, respectively. Although there is essentially only one parameter $\sigma$ (the coupling $\alpha_{S}$ is given by the one-loop result and the Debye mass is given by the HTL) for the case of no bulk viscous correction, the computed melting temperatures is in a reasonable agreement.

In figure 13, we plot the melting temperatures of the states $J / \psi, \Upsilon$ and $\Upsilon^{\prime}$ as a function of the bulk viscous parameter $\Phi$. The colored regions indicate the range between the values of $\Lambda, 2 \times 0.176 \mathrm{GeV}$ and $0.5 \times 0.176 \mathrm{GeV}$, and the solid line is for $\Lambda=0.176 \mathrm{GeV}$. We observe a mild decrease of the melting temperature as a function of $\Phi$. The slope of $\Upsilon^{\prime}$ is steeper compared to $J / \psi$ and $\Upsilon$. Therefore, we have found that the bulk viscous effect indeed affect the melting temperature, especially for excited states. When the plasma is close to the critical point, the bulk viscous contribution is expected to be enlarged. This can lead an anomalous behavior of observables related to heavy quarkonia in the beam energy scan program, although at this state it is difficult to make a quantitative estimate. In order to make a quantitative predictions, one should combine such behavior of quarkonia with a dynamical framework based on hydrodynamics.

\section{$5 \quad$ Summary and discussions}

Heavy quarkonia are useful in probing the nature of the medium around them, through the modification of their properties. In this article, we have studied how the non-equilibrium bulk viscous corrections are imprinted in the properties of heavy quarkonia. The bulk viscous correction modifies the distribution functions of thermal particles, from which the modified dielectric permittivity is computed in the HTL approximation. Using the dielectric permittivity, we computed the heavy quarkonia potential and examined how the potential is deformed in the presence of bulk viscous corrections. As for the real part of the potential, the bulk viscous correction parametrized by $\Phi>0$ leads to a larger Debye mass, hence stronger screening. The magnitude of the imaginary part is enhanced at short distances, and is suppressed at large $r$, as shown in figure 4. We have tried three prescriptions to accommodate the non-perturbative string-like potential at finite temperatures. Qualitative features of how the bulk viscosity affect the potential is found to be the same among different prescriptions, which indicates the robustness of the results. 
We solved the Schrödinger equation with the real part of the potential to obtain the deformed wave functions. We computed the binding energies and decay widths for $J / \psi, \psi^{\prime}$, $\Upsilon, \Upsilon^{\prime}$ states at different temperatures and the parameter $\Phi$ that quantify the bulk viscous corrections. Basically, a positive $\Phi$ leads to a Debye mass and it reduces the binding energy. On the other hand, the decay width is enhanced for a given temperature. Because of those, the melting temperature, at which the binding energy equals the decay width, is reduced for $\Phi>0$ and is enhanced for $\Phi<0$. When the system is near the critical point, the bulk viscous effect is expected to be enhanced, that would affect the melting temperature. It would be interesting to find an anomalous behavior of observables such as $R_{A A}$ in the beam energy scan program.

Finally, let us make several comments about the possible future directions. A unique ability of potential models is that it can be extended to non-equilibrium situations. It would be interesting to combine the method of potentials with the technique of the QCD sum rule [85-89] to gain insight into the properties of out-of-equilibrium QCD.

Technically and conceptually, in order to understand the dynamical evolution of heavy quarkonia as a quantum state, the analysis based on the theory of open quantum systems would be desirable, which is actively studied recently [90-97]. It would be interesting to apply/derive such a framework to non-equilibrium environments which can have anisotropic noises and see how the time evolution of quarkonia is affected.

Although we have demonstrated that heavy quarkonia have a potential to be sensitive to the bulk viscous nature of the surrounding media, how to experimentally probe this is a nontrivial question. Due to the non-equilibrium correction, the fluctuation-dissipation theorem is violated, which leads to two different Debye masses for retarded and symmetric propagators. If we can make an estimate of those two mass scales independently, it will be possible to explore the non-equilibrium bulk viscous corrections, that are expected to be significant near the critical point.

\section{Acknowledgments}

L.T. and Y.H. was funded by the Korean Ministry of Education, Science and Technology, Gyeongsangbuk-do and Pohang City at the Asia Pacific Center for Theoretical Physics (APCTP). N. H. was funded by Department of Atomic Energy (DAE), India via National Institute of Science Education and Research.

Open Access. This article is distributed under the terms of the Creative Commons Attribution License (CC-BY 4.0), which permits any use, distribution and reproduction in any medium, provided the original author(s) and source are credited.

\section{References}

[1] H.A. Weldon, Covariant calculations at finite temperature: the relativistic plasma, Phys. Rev. D 26 (1982) 1394 [INSPIRE].

[2] E. Braaten and R.D. Pisarski, Soft amplitudes in hot gauge theories: a general analysis, Nucl. Phys. B 337 (1990) 569 [inSPIRE].

[3] J. Frenkel and J.C. Taylor, High temperature limit of thermal QCD, Nucl. Phys. B 334 (1990) 199 [INSPIRE]. 
[4] E. Braaten and R.D. Pisarski, Simple effective lagrangian for hard thermal loops, Phys. Rev. D 45 (1992) R1827 [inSPIRE].

[5] J.O. Andersen, E. Braaten and M. Strickland, Hard thermal loop resummation of the free energy of a hot gluon plasma, Phys. Rev. Lett. 83 (1999) 2139 [hep-ph/9902327] [INSPIRE].

[6] J.O. Andersen, L.E. Leganger, M. Strickland and N. Su, Three-loop HTL QCD thermodynamics, JHEP 08 (2011) 053 [arXiv: 1103.2528] [INSPIRE].

[7] N. Haque et al., Three-loop HTLpt thermodynamics at finite temperature and chemical potential, JHEP 05 (2014) 027 [arXiv: 1402.6907] [INSPIRE].

[8] W. Lucha, F.F. Schoberl and D. Gromes, Bound states of quarks, Phys. Rept. 200 (1991) 127 [INSPIRE].

[9] N. Brambilla, A. Pineda, J. Soto and A. Vairo, Effective field theories for heavy quarkonium, Rev. Mod. Phys. 77 (2005) 1423 [hep-ph/0410047] [INSPIRE].

[10] E. Eichten et al., The spectrum of charmonium, Phys. Rev. Lett. 34 (1975) 369 [Erratum ibid. 36 (1976) 1276] [INSPIRE].

[11] E. Eichten et al., Charmonium: comparison with experiment, Phys. Rev. D 21 (1980) 203 [INSPIRE].

[12] T. Matsui and H. Satz, J/ $\psi$ Suppression by quark-Gluon plasma Formation, Phys. Lett. B 178 (1986) 416 [INSPIRE].

[13] F. Karsch, M.T. Mehr and H. Satz, Color screening and deconfinement for bound states of heavy quarks, Z. Phys. C 37 (1988) 617 [InSPIRE].

[14] A. Mócsy and P. Petreczky, Heavy quarkonia survival in potential model, Eur. Phys. J. C 43 (2005) 77 [hep-ph/0411262] [INSPIRE].

[15] C.-Y. Wong, Heavy quarkonia in quark-gluon plasma, Phys. Rev. C 72 (2005) 034906 [hep-ph/0408020] [INSPIRE].

[16] A. Mócsy and P. Petreczky, Quarkonia correlators above deconfinement, Phys. Rev. D 73 (2006) 074007 [hep-ph/0512156] [INSPIRE].

[17] D. Cabrera and R. Rapp, T-matrix approach to quarkonium correlation Functions in the QGP, Phys. Rev. D 76 (2007) 114506 [hep-ph/0611134] [INSPIRE].

[18] A. Mócsy and P. Petreczky, Color screening melts quarkonium, Phys. Rev. Lett. 99 (2007) 211602 [arXiv: 0706 .2183] [INSPIRE].

[19] W.M. Alberico, A. Beraudo, A. De Pace and A. Molinari, Potential models and lattice correlators for quarkonia at finite temperature, Phys. Rev. D 77 (2008) 017502 [arXiv:0706.2846] [INSPIRE].

[20] A. Mócsy , Potential models for quarkonia, Eur. Phys. J. C 61 (2009) 705 [arXiv:0811.0337] [INSPIRE].

[21] F. Karsch, M.G. Mustafa and M.H. Thoma, Finite temperature meson correlation functions in HTL approximation, Phys. Lett. B 497 (2001) 249 [hep-ph/0007093] [INSPIRE].

[22] T. Umeda, K. Nomura and H. Matsufuru, Charmonium at finite temperature in quenched lattice QCD, Eur. Phys. J. C 39S1 (2005) 9 [hep-lat/0211003] [InSPIRE].

[23] M. Asakawa and T. Hatsuda, $J / \psi$ and $\eta_{c}$ in the deconfined plasma from lattice QCD, Phys. Rev. Lett. 92 (2004) 012001 [hep-lat/0308034] [INSPIRE].

[24] S. Datta, F. Karsch, P. Petreczky and I. Wetzorke, Behavior of charmonium systems after deconfinement, Phys. Rev. D 69 (2004) 094507 [hep-lat/0312037] [INSPIRE]. 
[25] G. Aarts et al., Charmonium at high temperature in two-flavor QCD, Phys. Rev. D 76 (2007) 094513 [arXiv:0705.2198] [INSPIRE].

[26] A. Jakovac, P. Petreczky, K. Petrov and A. Velytsky, Quarkonium correlators and spectral functions at zero and finite temperature, Phys. Rev. D 75 (2007) 014506 [hep-lat/0611017] [INSPIRE].

[27] H.T. Ding et al., Charmonium properties in hot quenched lattice QCD, Phys. Rev. D 86 (2012) 014509 [arXiv: 1204.4945] [INSPIRE].

[28] WHOT-QCD collaboration, Charmonium spectral functions with the variational method in zero and finite temperature lattice QCD, Phys. Rev. D 84 (2011) 094504 [arXiv:1104.3384] [INSPIRE].

[29] R. Larsen, S. Meinel, S. Mukherjee and P. Petreczky, Excited bottomonia in quark-gluon plasma from lattice QCD, Phys. Lett. B 800 (2020) 135119 [arXiv:1910.07374].

[30] R. Larsen, S. Meinel, S. Mukherjee and P. Petreczky, Thermal broadening of bottomonia: lattice nonrelativistic QCD with extended operators, Phys. Rev. D 100 (2019) 074506 [arXiv: 1908.08437] [INSPIRE].

[31] M. Laine, O. Philipsen, P. Romatschke and M. Tassler, Real-time static potential in hot QCD, JHEP 03 (2007) 054 [hep-ph/0611300] [INSPIRE].

[32] M. Laine, O. Philipsen and M. Tassler, Thermal imaginary part of a real-time static potential from classical lattice gauge theory simulations, JHEP 09 (2007) 066 [arXiv:0707.2458] [INSPIRE].

[33] Y. Burnier, M. Laine and M. Vepsäläinen, Heavy quarkonium in any channel in resummed hot QCD, JHEP 01 (2008) 043 [arXiv:0711.1743] [INSPIRE].

[34] A. Beraudo, J.P. Blaizot and C. Ratti, Real and imaginary-time $Q \bar{Q}$ correlators in a thermal medium, Nucl. Phys. A 806 (2008) 312 [arXiv:0712.4394] [INSPIRE].

[35] N. Brambilla, J. Ghiglieri, A. Vairo and P. Petreczky, Static quark-antiquark pairs at finite temperature, Phys. Rev. D 78 (2008) 014017 [arXiv:0804.0993] [INSPIRE].

[36] N. Brambilla, M.A. Escobedo, J. Ghiglieri and A. Vairo, Thermal width and gluo-dissociation of quarkonium in pNRQCD, JHEP 12 (2011) 116 [arXiv:1109.5826] [INSPIRE].

[37] N. Brambilla, M.A. Escobedo, J. Ghiglieri and A. Vairo, Thermal width and quarkonium dissociation by inelastic parton scattering, JHEP 05 (2013) 130 [arXiv:1303.6097] [INSPIRE].

[38] A. Dumitru, Y. Guo and M. Strickland, The imaginary part of the static gluon propagator in an anisotropic (viscous) QCD plasma, Phys. Rev. D 79 (2009) 114003 [arXiv:0903.4703] [INSPIRE].

[39] P. Petreczky, C. Miao and A. Mócsy, Quarkonium spectral functions with complex potential, Nucl. Phys. A 855 (2011) 125 [arXiv: 1012.4433] [InSPIRE].

[40] M. Margotta et al., Quarkonium states in a complex-valued potential, Phys. Rev. D 83 (2011) 105019 [Erratum ibid. D 84 (2011) 069902] [arXiv:1101.4651] [INSPIRE].

[41] L. Thakur, U. Kakade and B.K. Patra, Dissociation of quarkonium in a Complex potential, Phys. Rev. D 89 (2014) 094020 [arXiv: 1401.0172] [INSPIRE].

[42] A. Rothkopf, T. Hatsuda and S. Sasaki, Complex heavy-quark potential at finite temperature from lattice QCD, Phys. Rev. Lett. 108 (2012) 162001 [arXiv:1108.1579] [INSPIRE].

[43] Y. Burnier and A. Rothkopf, Disentangling the timescales behind the non-perturbative heavy quark potential, Phys. Rev. D 86 (2012) 051503 [arXiv:1208.1899] [INSPIRE]. 
[44] Y. Burnier and A. Rothkopf, A gauge invariant Debye mass and the complex heavy-quark potential, Phys. Lett. B 753 (2016) 232 [arXiv:1506.08684] [INSPIRE].

[45] A. Rothkopf, Heavy quarkonium in extreme conditions, Phys. Rept. 858 (2020) 1 [arXiv: 1912.02253] [INSPIRE].

[46] A. Dumitru, Y. Guo, A. Mócsy and M. Strickland, Quarkonium states in an anisotropic QCD plasma, Phys. Rev. D 79 (2009) 054019 [arXiv:0901.1998] [InSPIRE].

[47] O. Philipsen and M. Tassler, On quarkonium in an anisotropic quark gluon plasma, arXiv:0908.1746 [INSPIRE].

[48] A. Dumitru, Quarkonium in a non-ideal hot QCD plasma, Prog. Theor. Phys. Suppl. 187 (2011) 87 [arXiv: 1010.5218] [inSPIRE].

[49] L. Thakur, N. Haque, U. Kakade and B.K. Patra, Dissociation of quarkonium in an anisotropic hot QCD medium, Phys. Rev. D 88 (2013) 054022 [arXiv:1212.2803] [INSPIRE].

[50] M. Strickland and D. Bazow, Thermal bottomonium suppression at RHIC and LHC, Nucl. Phys. A 879 (2012) 25 [arXiv:1112.2761] [INSPIRE].

[51] M. Strickland, Thermalization and isotropization in heavy-ion collisions, Pramana 84 (2015) 671 [arXiv: 1312.2285] [INSPIRE].

[52] M. Strickland, Anisotropic hydrodynamics: three lectures, Acta Phys. Polon. B 45 (2014) 2355 [arXiv: 1410.5786] [inSPIRE].

[53] C. Bonati, M. D'Elia and A. Rucci, Heavy quarkonia in strong magnetic fields, Phys. Rev. D 92 (2015) 054014 [arXiv: 1506.07890] [INSPIRE].

[54] C. Bonati et al., Magnetic field effects on the static quark potential at zero and finite temperature, Phys. Rev. D 94 (2016) 094007 [arXiv: 1607.08160] [INSPIRE].

[55] K. Marasinghe and K. Tuchin, Quarkonium dissociation in quark-gluon plasma via ionization in magnetic field, Phys. Rev. C 84 (2011) 044908 [arXiv:1103.1329] [INSPIRE].

[56] J. Alford and M. Strickland, Charmonia and bottomonia in a magnetic field, Phys. Rev. D 88 (2013) 105017 [arXiv: 1309.3003] [INSPIRE].

[57] B. Singh, L. Thakur and H. Mishra, Heavy quark complex potential in a strongly magnetized hot QGP medium, Phys. Rev. D 97 (2018) 096011 [arXiv:1711.03071] [INSPIRE].

[58] M. Hasan, B. Chatterjee and B.K. Patra, Heavy quark potential in a static and strong homogeneous magnetic field, Eur. Phys. J. C 77 (2017) 767 [arXiv:1703.10508] [INSPIRE].

[59] J.C.S. Amal et al., Charmonium states in strong magnetic fields, Phys. Rev. C 98 (2018) 065202 [arXiv: 1803.04322] [INSPIRE].

[60] M. Kurian and V. Chandra, Bulk viscosity of a hot QCD medium in a strong magnetic field within the relaxation-time approximation, Phys. Rev. D 97 (2018) 116008 [arXiv: 1802.07904] [INSPIRE].

[61] M. Kurian, V. Chandra and S.K. Das, Impact of longitudinal bulk viscous effects to heavy quark transport in a strongly magnetized hot QCD medium, Phys. Rev. D 101 (2020) 094024 [arXiv: 2002.03325] [INSPIRE].

[62] M.A. Escobedo, J. Soto and M. Mannarelli, Non-relativistic bound states in a moving thermal bath, Phys. Rev. D 84 (2011) 016008 [arXiv:1105.1249] [InSPIRE].

[63] M.A. Escobedo, F. Giannuzzi, M. Mannarelli and J. Soto, Heavy quarkonium moving in a quark-gluon plasma, Phys. Rev. D 87 (2013) 114005 [arXiv:1304.4087] [INSPIRE].

[64] L. Thakur, N. Haque and H. Mishra, Heavy quarkonium moving in hot and dense deconfined nuclear matter, Phys. Rev. D 95 (2017) 036014 [arXiv:1611.04568] [INSPIRE]. 
[65] S.D. Avramis, K. Sfetsos and D. Zoakos, On the velocity and chemical-potential dependence of the heavy-quark interaction in N=4 SYM plasmas, Phys. Rev. D 75 (2007) 025009 [hep-th/0609079] [INSPIRE].

[66] Y. Liu, N. Xu and P. Zhuang, Velocity dependence of charmonium dissociation temperature in high-energy nuclear collisions, Phys. Lett. B 724 (2013) 73 [arXiv:1210.7449] [INSPIRE].

[67] M. Ali-Akbari, D. Giataganas and Z. Rezaei, Imaginary potential of heavy quarkonia moving in strongly coupled plasma, Phys. Rev. D 90 (2014) 086001 [arXiv:1406.1994] [InSPIRE].

[68] B.K. Patra, H. Khanchandani and L. Thakur, Velocity-induced heavy quarkonium dissociation using the gauge-gravity correspondence, Phys. Rev. D 92 (2015) 085034 [arXiv: 1504.05396] [INSPIRE].

[69] D. Lafferty and A. Rothkopf, Improved Gauss law model and in-medium heavy quarkonium at finite density and velocity, Phys. Rev. D 101 (2020) 056010 [arXiv:1906.00035] [INSPIRE].

[70] A. Bzdak et al., Mapping the phases of quantum chromodynamics with beam energy scan, Phys. Rept. 853 (2020) 1 [arXiv:1906.00936] [INSPIRE].

[71] D. Kharzeev and K. Tuchin, Bulk viscosity of QCD matter near the critical temperature, JHEP 09 (2008) 093 [arXiv:0705.4280] [INSPIRE].

[72] F. Karsch, D. Kharzeev and K. Tuchin, Universal properties of bulk viscosity near the QCD phase transition, Phys. Lett. B 663 (2008) 217 [arXiv:0711.0914] [inSPIRE].

[73] G.D. Moore and O. Saremi, Bulk viscosity and spectral functions in QCD, JHEP 09 (2008) 015 [arXiv: 0805.4201] [INSPIRE].

[74] Q. Du, A. Dumitru, Y. Guo and M. Strickland, Bulk viscous corrections to screening and damping in QCD at high temperatures, JHEP 01 (2017) 123 [arXiv:1611.08379] [INSPIRE].

[75] M. Cheng et al., The spatial string tension and dimensional reduction in QCD, Phys. Rev. D 78 (2008) 034506 [arXiv:0806.3264] [INSPIRE].

[76] WHOT-QCD collaboration, Heavy-quark free energy, debye mass and spatial string tension at finite temperature in two flavor lattice $Q C D$ with Wilson quark action, Phys. Rev. D 75 (2007) 074501 [hep-lat/0702004] [INSPIRE].

[77] O. Andreev and V.I. Zakharov, The spatial string tension, thermal phase transition and AdS/QCD, Phys. Lett. B 645 (2007) 437 [hep-ph/0607026] [INSPIRE].

[78] V. Agotiya, V. Chandra and B.K. Patra, Dissociation of quarkonium in hot QCD medium: modification of the inter-quark potential, Phys. Rev. C 80 (2009) 025210 [arXiv:0808.2699] [INSPIRE].

[79] Y. Guo, L. Dong, J. Pan and M.R. Moldes, Modelling the non-perturbative contributions to the complex heavy-quark potential, Phys. Rev. D 100 (2019) 036011 [arXiv:1806.04376] [INSPIRE].

[80] S. Mrowczynski and M.H. Thoma, Hard loop approach to anisotropic systems, Phys. Rev. D 62 (2000) 036011 [hep-ph/0001164] [INSPIRE].

[81] G.S. Denicol, W. Florkowski, R. Ryblewski and M. Strickland, Shear-bulk coupling in nonconformal hydrodynamics, Phys. Rev. C 90 (2014) 044905 [arXiv: 1407.4767] [INSPIRE].

[82] M.E. Carrington, D.-f. Hou and M.H. Thoma, Equilibrium and nonequilibrium hard thermal loop resummation in the real time formalism, Eur. Phys. J. C 7 (1999) 347 [hep-ph/9708363] [INSPIRE].

[83] E. Megias, E. Ruiz Arriola and L.L. Salcedo, Dimension two condensates and the Polyakov loop above the deconfinement phase transition, JHEP 01 (2006) 073 [hep-ph/0505215] [INSPIRE]. 
[84] A. Bazavov et al., Determination of $\alpha_{s}$ from the QCD static energy, Phys. Rev. D 86 (2012) 114031 [arXiv: 1205.6155] [INSPIRE].

[85] K. Morita and S.H. Lee, Heavy quarkonium correlators at finite temperature: QCD sum rule approach, Phys. Rev. D 82 (2010) 054008 [arXiv:0908.2856] [INSPIRE].

[86] K. Morita and S.H. Lee, Mass shift and width broadening of $J / \psi$ in $Q G P$ from $Q C D$ sum rule, Phys. Rev. Lett. 100 (2008) 022301 [arXiv:0704.2021] [INSPIRE].

[87] P. Gubler, K. Morita and M. Oka, Charmonium spectra at finite temperature from QCD sum rules with the maximum entropy method, Phys. Rev. Lett. 107 (2011) 092003 [arXiv: 1104.4436] [INSPIRE].

[88] K.-J. Araki, K. Suzuki, P. Gubler and M. Oka, Charmonium ground and excited states at finite temperature from complex Borel sum rules, Phys. Lett. B 780 (2018) 48 [arXiv: 1703.01438] [INSPIRE].

[89] P. Gubler and D. Satow, Recent progress in QCD condensate evaluations and sum rules, Prog. Part. Nucl. Phys. 106 (2019) 1 [arXiv:1812.00385] [INSPIRE].

[90] C. Young and K. Dusling, Quarkonium above deconfinement as an open quantum system, Phys. Rev. C 87 (2013) 065206 [arXiv: 1001.0935] [INSPIRE].

[91] Y. Akamatsu and A. Rothkopf, Stochastic potential and quantum decoherence of heavy quarkonium in the quark-gluon plasma, Phys. Rev. D 85 (2012) 105011 [arXiv:1110.1203] [INSPIRE].

[92] Y. Akamatsu, Heavy quark master equations in the Lindblad form at high temperatures, Phys. Rev. D 91 (2015) 056002 [arXiv:1403.5783] [InSPIRE].

[93] J.-P. Blaizot, D. De Boni, P. Faccioli and G. Garberoglio, Heavy quark bound states in a quark-gluon plasma: dissociation and recombination, Nucl. Phys. A 946 (2016) 49 [arXiv: 1503.03857] [INSPIRE].

[94] D. De Boni, Fate of in-medium heavy quarks via a Lindblad equation, JHEP 08 (2017) 064 [arXiv: 1705.03567] [INSPIRE].

[95] S. Kajimoto, Y. Akamatsu, M. Asakawa and A. Rothkopf, Dynamical dissociation of quarkonia by wave function decoherence, Phys. Rev. D 97 (2018) 014003 [arXiv: 1705.03365] [INSPIRE].

[96] N. Brambilla, M.A. Escobedo, J. Soto and A. Vairo, Heavy quarkonium suppression in a fireball, Phys. Rev. D 97 (2018) 074009 [arXiv:1711.04515] [INSPIRE].

[97] Y. Akamatsu, M. Asakawa, S. Kajimoto and A. Rothkopf, Quantum dissipation of a heavy quark from a nonlinear stochastic Schrödinger equation, JHEP 07 (2018) 029 [arXiv: 1805.00167] [INSPIRE]. 